Pacific

Journal of

Mathematics

UNIFORM INTEGRABILITY OF APPROXIMATE GREEN FUNCTIONS OF SOME DEGENERATE ELLIPTIC OPERATORS

Ahmed Mohammed 


\title{
UNIFORM INTEGRABILITY OF APPROXIMATE GREEN FUNCTIONS OF SOME DEGENERATE ELLIPTIC OPERATORS
}

\author{
Ahmed Mohammed
}

We prove the uniform integrability of the approximate Green functions of some degenerate elliptic operators in divergence form with lower order term coefficients satisfying a Kato type condition. Some further properties of the approximate Green functions of such operators are also established.

\section{Introduction.}

In this paper, we study the approximate Green functions of certain degenerate elliptic operators $L$ on balls in $\mathbb{R}^{n}, n>2$, when $L$ has the divergence form

$$
L:=-\sum_{i, j=1}^{n} \frac{\partial}{\partial x_{i}}\left(a_{i j}(x) \frac{\partial}{\partial x_{j}}\right)+\sum_{i=1}^{n} b_{i}(x) \frac{\partial}{\partial x_{i}}+V(x) .
$$

The coefficients $a_{i j}$ are real-valued measurable functions whose coefficient matrix $A(x):=\left(a_{i j}(x)\right)$ is symmetric and satisfies

$$
\omega(x)|\xi|^{2} \leq\langle A(x) \xi, \xi\rangle \leq v(x)|\xi|^{2} .
$$

Here $\langle.,$.$\rangle denotes the usual Euclidean inner product, and v, \omega$ are weight functions that will be stipulated below.

Throughout, we will use the following notations. For functions $f$ and $g$, we shall write $f \lesssim g$ to indicate that $f \leq C g$ for some positive constant $C$. We write $f \approx g$ if $f \lesssim g$ and $g \lesssim f$. We shall use $B_{t}(x)$ to designate a ball of radius $t$ centered at $x$. Also, $t B$ will be used to represent the ball concentric with the ball $B$, but with radius $t$ times as big. Given a locally integrable function $f$, we shall let $f(B)$ denote the Lebesgue integral of $f$ over the set $B$. If $f \in L_{l o c}(d \mu)$, where $d \mu:=\gamma(x) d x$ is a weighted measure, then we denote by

$$
f_{B} f(x) \gamma(x) d x:=\frac{1}{\gamma(B)} \int_{B} f(x) \gamma(x) d x,
$$

the $\mu$-average of $f$ over $B$. This average shall also be denoted by $f_{B}, \gamma$. 
A non-negative locally integrable function $\omega$ on $\mathbb{R}^{n}$ is said to be in the class $A_{2}$ if $1 / \omega$ is also locally integrable and there is a constant $C$ such that for all balls $B$,

$$
\left(f_{B} \omega(x) d x\right)\left(f_{B} \frac{1}{\omega(x)} d x\right) \leq C .
$$

A non-negative locally integrable function $v$ on $\mathbb{R}^{n}$ is said to satisfy a doubling condition if there is a constant $C$ such that $v(2 B) \leq C v(B)$ for all balls $B$. Here $C$ is independent of the center and radius of $B$. We denote this by writing $v \in D_{\infty}$. It is known that $A_{2} \subset D_{\infty}$.

It is also known (see [9]) that if $v$ satisfies a doubling condition, then it satisfies

$$
v(t B) \leq C_{1} t^{k} v(B), \text { and } v(B) \leq C_{2} t^{-m}(t B), \quad t>1,
$$

for some positive constants $C_{1}, C_{2}, k$, and $m$. The second condition is called a reverse doubling condition.

The following assumptions will be made on $\omega$, and $v$.

$\omega$ and $v$ are non-negative locally integrable functions on $\mathbb{R}^{n}$ that satisfy the following conditions:

$$
\begin{aligned}
& \omega \in A_{2}, v \in D_{\infty} ; \\
& \omega \text { and } v \text { are related by the existence of some } q>2 \text { such that }
\end{aligned}
$$

$$
\frac{s}{t}\left[\frac{v\left(B_{s}(x)\right)}{v\left(B_{t}(x)\right)}\right]^{\frac{1}{q}} \leq C\left[\frac{\omega\left(B_{s}(x)\right)}{\omega\left(B_{t}(x)\right)}\right]^{\frac{1}{2}}, \quad 0<s<t, \quad x \in \mathbb{R}^{n},
$$

for some constant $C$ independent of $x, s$ and $t$.

We shall use the notation $\sigma=q / 2$ so that $\sigma>1$. Note that when $v$ and $\omega$ are positive constants, as in the strongly elliptic case, the value of $q$ in (1.3) is $q=2 n /(n-2)$, so that $\sigma=n /(n-2)$.

Let now $L_{0}$ be the principal part of $L$; that is

$$
L_{0}:=-\sum_{i, j=1}^{n} \frac{\partial}{\partial x_{i}}\left(a_{i j}(x) \frac{\partial}{\partial x_{j}}\right) .
$$

Let $B_{0}$ be a ball of radius $R$ that will be fixed in the sequel. Under the conditions (1.2) and (1.3), Chanillo and Wheeden have established, in [3] the existence and integrability properties of the Green function of $L_{0}$. Among other important properties, they have shown that if $G(x, y)$ is the Green function of $L_{0}$ on $2 B_{0}$, then for $0<p<\sigma$,

$$
\sup _{y \in B_{0}} \int_{2 B_{0}} G(x, y)^{p} v(x) d x<\infty .
$$


Let $B \subset B_{0}$. In analogy with the way the usual Kato class is defined, we introduce a class of functions $K^{n}(B)$ as

$$
K^{n}(B):=\left\{h \in L_{\mathrm{loc}}^{1}(B): \lim _{r \rightarrow 0^{+}} \eta(h)(r)=0\right\},
$$

where

$$
\eta(h)(r):=\sup _{x \in B} \int_{B_{r}(x) \cap B} G(y, x)|h(y)| d y .
$$

If $L_{\mu}^{p}(B)$ denotes the usual $L^{p}$ space with respect to the measure $\mu$, then for $B \subset B_{0}$, and $p>\sigma /(\sigma-1)$, the following inclusion holds:

$$
L_{v^{1-p}}^{p}(B) \subset K^{n}(B) .
$$

To see this let $h \in L_{v^{1-p}}^{p}(B)$, and $x \in B$. We pick $\sigma /(\sigma-1)<s<p$. Define $s^{\prime}$ by $1 / s+1 / s^{\prime}=1$ (we will use this notation throughout). Then, by Hölder inequality

$$
\begin{aligned}
\int_{B_{r}(x) \cap B} G(y, x)|h(y)| d y \leq & \left(\int_{2 B_{0}} G(y, x)^{s^{\prime}} v(y) d y\right)^{\frac{1}{s^{\prime}}} \\
& \cdot\left(\int_{B_{r}(x) \cap B}|h(y)|^{s} v^{1-s} d y\right)^{\frac{1}{s}} .
\end{aligned}
$$

Since $v$ satisfies a reverse doubling condition, there exist positive constants $C$ and $d$ such that $v\left(B_{R}(x)\right) \geq C(R / r)^{d} v\left(B_{r}(x)\right)$ for any $0<r<R$. Therefore

$$
\begin{aligned}
& \int_{B_{r}(x) \cap B}\left(\frac{|h(y)|}{v}\right)^{s} v d x \\
& \leq\left(\int_{B_{r}(x) \cap B}\left(\frac{|h(y)|}{v}\right)^{p} v d x\right)^{\frac{s}{p}}\left(\int_{B_{r}(x)} v d x\right)^{\frac{p-s}{p}} \\
& \leq\left(\int_{B}\left(\frac{|h(y)|}{v}\right)^{p} v d x\right)^{\frac{s}{p}}\left[C\left(\frac{r}{R}\right)^{d}\right]^{\frac{p-s}{p}}\left[v\left(2 B_{0}\right)\right]^{\frac{p-s}{p}} .
\end{aligned}
$$

Thus, from this last inequality and (1.4), we get the desired conclusion.

For notational simplicity, we shall use $K$ for the function space $K^{n}\left(B_{0}\right)$.

Remark 1.1. We should remark that when $v$ and $\omega$ are identically equal to positive constants, as in the strongly elliptic case, the class of functions $K$ coincides with the usual Kato class (see [1], or [4] for definition). Also, if $v$ and $\omega$ are constant multiples of each other, then again $K$ is the same as the one introduced in [6].

The following assumptions will be made of the lower order coefficients $\mathbf{b}:=\left(b_{1}, b_{2}, \cdots, b_{n}\right)$, and $V$ of the degenerate elliptic operator $L$.

$$
|\mathbf{b}|^{2} \omega^{-1}, V \in K \text {. }
$$


The paper is organized as follows. As the work here relies heavily on the results of the important works of S. Chanillo and R. Wheeden in their papers $[\mathbf{2}]$, and $[\mathbf{3}]$, we will recall several of their results that are relevant to our discussion in Section 2. We start Section 3 by proving the boundedness of certain linear functionals on some Hilbert spaces. These functionals are associated with elements of the Kato type class defined above. Some properties related to the approximate Green function of $L_{0}$ will also be obtained. The main result in this paper is Theorem 3.2 which establishes the uniform integrability of approximate Green functions of $L$ on balls. Uniform integrability of approximate Green functions is a useful tool in proving existence and size estimates of the Green function. See $[\mathbf{3}],[5]$ and $[\mathbf{8}]$ for such applications. In a forthcoming paper, we will use this uniform integrability result to derive Harnack's inequality for functions naturally associated with non-negative solutions of the operator $L$.

\section{Preliminaries and background.}

Let $\Omega \subset \mathbb{R}^{n}$ be a bounded open set. Using a standard notation, let $\operatorname{Lip}(\bar{\Omega})$ denote the class of Lipschitz continuous functions on the closure $\bar{\Omega}$. We say that $\phi \in \operatorname{Lip}_{0}(\Omega)$ if $\phi \in \operatorname{Lip}(\bar{\Omega})$ and $\phi$ has compact support contained in $\Omega$. The following two-weight Sobolev inequality has been proved in [2].

Let $\omega, v$ be non-negative locally integrable functions that satisfy (1.2), (1.3), and $q$ be the constant that appears in (1.3). Then, for any ball $B$,

$$
\left(f_{B}|f|^{q} v d x\right)^{\frac{1}{q}} \leq C|B|^{\frac{1}{n}}\left(f_{B}|\nabla f|^{2} \omega d x\right)^{\frac{1}{2}}, f \in \operatorname{Lip}_{0}(B) .
$$

The constant $C$ is independent of both the ball $B$ and $f$.

Now let us consider the inner product

$$
a_{0}(u, \varphi):=\int_{\Omega}\langle A \nabla u, \nabla \varphi\rangle, \quad u, \varphi \in \operatorname{Lip}_{0}(\Omega) .
$$

The completion of $\operatorname{Lip}_{0}(\Omega)$ with respect to the norm $a_{0}(u, u)^{1 / 2}$ is denoted by $H_{0}(\Omega)$. An element of $H_{0}(\Omega)$ is thus an equivalence class of Cauchy sequences $\left\{u_{k}\right\}, u_{k} \in \operatorname{Lip}_{0}(\Omega)$. If $u, \varphi \in H_{0}(\Omega)$, with $u=\left\{u_{k}\right\}, \varphi=$ $\left\{\varphi_{k}\right\}, u_{k}, \varphi_{k} \in \operatorname{Lip}_{0}(\Omega)$, then $a_{0}\left(u_{k}, \varphi_{k}\right)$ is convergent, and we define

$$
a_{0}(u, \varphi)=\lim _{k} a_{0}\left(u_{k}, \varphi_{k}\right) .
$$

In this way, $\|u\|_{0}:=a_{0}(u, u)^{1 / 2}$ defines a norm on the Hilbert space $H_{0}(\Omega)$. $\operatorname{Lip}_{0}(B)$ is included in $H_{0}(B)$ by considering $\left\{\varphi_{k}\right\}$ with all $\varphi_{k}=\varphi \in$ $\operatorname{Lip}_{0}(B)$. As a consequence of the Sobolev inequality (2.1), it is possible to associate with each $\varphi \in H_{0}(\Omega)$ a unique pair $(\tilde{\varphi}, \nabla \tilde{\varphi})$ so that if $\varphi=\left\{\varphi_{k}\right\}$, then $\varphi_{k} \rightarrow \tilde{\varphi}$ in $L_{v}^{2 \sigma}(\Omega)$, and $\nabla \varphi_{k} \rightarrow \nabla \tilde{\varphi}$ in $L_{\omega}^{2}(\Omega)$. We shall refer to $(\tilde{\varphi}, \nabla \tilde{\varphi})$ 
as the pair of functions associated with $\varphi$. This pair is independent of the particular representation $\left\{\varphi_{k}\right\}$ of $\varphi$. If $\varphi \in \operatorname{Lip}_{0}(\Omega)$, then $\tilde{\varphi}=\varphi$, and $\nabla \tilde{\varphi}=\nabla \varphi$. Furthermore, it can be shown that given $\varphi \in H_{0}(\Omega), \nabla \tilde{\varphi}$ is the distributional gradient of $\tilde{\varphi}$. See $[\mathbf{3}]$ for proofs of these assertions.

For future reference, we record the following inequality that can be easily verified using the Cauchy-Schwarz inequality.

$$
\|\varphi \phi\|_{0} \leq\|\varphi\|_{\infty}\|\phi\|_{0}+\|\phi\|_{\infty}\|\varphi\|_{0}, \quad \varphi, \phi \in \operatorname{Lip}_{0}(\Omega) .
$$

We will also consider the Hilbert space $H(\Omega)$ which is the completion of $\operatorname{Lip}(\bar{\Omega})$ under the inner product

$$
a(u, \varphi):=a_{0}(u, \varphi)+\int_{\Omega} u \varphi v, \quad u, \varphi \in \operatorname{Lip}(\bar{\Omega}) .
$$

If $u \in H(\Omega), u=\left\{u_{k}\right\}, u_{k} \in \operatorname{Lip}(\bar{\Omega})$, then $u_{k}$ converges in $L_{v}^{2}(\Omega)$ to a function $\tilde{u}$, and $\nabla u_{k}$ converges in $L_{\omega}^{2}(\Omega)$ to a vector $\nabla \tilde{u}$. If $\varphi=$ $\left\{\varphi_{k}\right\}, \varphi_{k} \in \operatorname{Lip}(\bar{\Omega})$, then the limits $a(u, \varphi)=\lim _{k} a\left(u_{k}, \varphi_{k}\right)$ and $a_{0}(u, \varphi)=$ $\lim _{k} a_{0}\left(u_{k}, \varphi_{k}\right)$ exist, and satisfy

$$
a(u, \varphi):=a_{0}(u, \varphi)+\int_{\Omega} \tilde{u} \tilde{\varphi} v
$$

In this way, $a(u, \varphi)$ defines an inner product on $H(\Omega)$, and $\|u\|:=a(u, u)^{1 / 2}$ defines a norm. By the Sobolev inequality $(2.1), H_{0}(\Omega)$ is continuously embedded in $H(\Omega)$.

For $u \in H(\Omega)$ we say that $u \geq 0$ on $\Omega$, if $u_{k} \geq 0$ for all $k$ and some $\left\{u_{k}\right\}$ representing $u$. If $u \geq 0$ on $\Omega$, then $\tilde{u} \geq 0$ a.e. on $\Omega$. The following, proved in [3], will be useful to us.

Let $u, \varphi \in H(\Omega)$, and $\nabla \tilde{u}, \nabla \tilde{\varphi}$ be the associated gradients respectively. If $u=\left\{u_{k}\right\}, \varphi=\left\{\varphi_{k}\right\}$, then as $k \rightarrow \infty$

$$
\int_{\Omega}\left|\left\langle A \nabla u_{k}, \nabla \varphi_{k}\right\rangle-\langle A \nabla \tilde{u}, \nabla \tilde{\varphi}\rangle\right| \rightarrow 0 .
$$

In particular

$$
a_{0}(u, \varphi)=\int_{\Omega}\langle A \nabla \tilde{u}, \nabla \tilde{\varphi}\rangle, \quad \text { and } a(u, \varphi)=\int_{\Omega}\langle A \nabla \tilde{u}, \nabla \tilde{\varphi}\rangle+\int_{\Omega} \tilde{u} \tilde{\varphi} v .
$$

Before we proceed further, we should perhaps make two remarks. Let $B \subset B_{0}$ be a ball.

Remark 2.1. If $f v^{-1} \in L_{v}^{(2 \sigma)^{\prime}}(B)$, then

$$
\varphi \mapsto \int_{B} f \tilde{\varphi}
$$

defines a continuous linear functional on $H_{0}(B)$. This follows from Hölder's inequality and the Sobolev inequality (2.1). Therefore, by the Lax-Milgram 
theorem there is a unique $u \in H_{0}(B)$ such that

$$
a_{0}(u, \varphi)=\int_{B} f \tilde{\varphi}
$$

We shall refer to $u$ as the Lax-Milgram solution of $L_{0} u=f$ in $B$ and $u=$ 0 on $\partial B$.

By the above Remark, given $x \in B$, and $\rho>0$ with $B_{\rho}(x) \subset B$ there is a unique $G^{\rho} \in H_{0}(B)$ such that

$$
a_{0}\left(G^{\rho}, \varphi\right)=f_{B_{\rho}(x)} \tilde{\varphi} v, \quad \varphi \in H_{0}(B) .
$$

$\tilde{G}^{\rho}$ is called an approximate Green's function of $L_{0}$ on $B$ with pole $x$.

Remark 2.2. Let $f \in L^{1}(B)$ such that the map in (2.4) is a continuous linear functional on $H_{0}(B)$. Suppose $\left\{\varphi_{k}\right\}$ is a bounded sequence in $H_{0}(B)$. Then $\left\{\varphi_{k}\right\}$ contains a subsequence $\left\{\varphi_{k_{j}}\right\}$ that converges weakly to some element $\varphi \in H_{0}(B)$. Now, if $u=\left\{u_{k}\right\} \in H(B)$ is fixed, then since $\left|a_{0}(u, \varphi)\right| \leq\|u\|\|\varphi\|_{0}$, we have

$$
\begin{aligned}
a_{0}(u, \varphi)=\lim a_{0}\left(u, \varphi_{k_{j}}\right) & =\lim \left[a_{0}\left(u_{k_{j}}, \varphi_{k_{j}}\right)+a_{0}\left(u-u_{k_{j}}, \varphi_{k_{j}}\right)\right] \\
& =\lim a_{0}\left(u_{k_{j}}, \varphi_{k_{j}}\right) .
\end{aligned}
$$

Therefore

$$
a_{0}(u, \varphi)-\int_{B} f \tilde{\varphi}=\lim _{j \rightarrow \infty}\left[a_{0}\left(u_{k_{j}}, \varphi_{k_{j}}\right)-\int_{B} f \tilde{\varphi}_{k_{j}}\right] .
$$

We shall need several lemmas from [3], and we will state them below for the readers' convenience.

Lemma 2.1. Suppose $u$ is a supersolution in $H_{0}(\Omega)$; that is $u \in H_{0}(\Omega)$, and $a_{0}(u, \varphi) \geq 0$ whenever $0 \leq \varphi \in \operatorname{Lip}_{0}(B)$. Then $u \geq 0$.

Proof. The proof that the approximate Green function $G^{\rho}$ of $L_{0}$ is nonnegative is given on page 323 of [3]. It depends on properties of the inner product $a_{0}(.,$.$) and the fact that a_{0}\left(G^{\rho}, \varphi\right) \geq 0$ for $0 \leq \varphi \in H_{0}(\Omega)$. Exactly the same proof applies in our case. In fact, if $u:=\left\{u_{k}\right\}$, then also $u=\left\{\left|u_{k}\right|\right\}$. See Lemma 3.6 below for a detailed proof.

Lemma 2.2. Let $G(x, y)$ be the Green function of $L_{0}$ on $2 B$ and $f v^{-1} \in$ $L_{v}^{t^{\prime}}(2 B)$ for some $t<\sigma$. If $u$ is the Lax-Milgram solution of $L_{0} u=f$ in $2 B$, then

$$
\tilde{u}(y)=\int_{2 B} G(x, y) f(x) d x, \quad \text { for a.e. } y \in B \text {. }
$$

Another useful Lemma is the following weak maximum principle (cf. Lemma 2.1 above). 
Lemma 2.3 (Weak Maximum Principle). Let $u \in H(\Omega)$ satisfy $a_{0}(u, \varphi) \geq$ 0 if $\varphi \in \operatorname{Lip}_{0}(\Omega), \varphi \geq 0$. Let $u=\left\{u_{k}\right\}, u_{k} \in \operatorname{Lip}(\bar{\Omega})$ and assume that $u_{k} \geq 0$ in some neighborhood (depending on $k$ ) of $\partial \Omega$. Then $\tilde{u} \geq 0$ a.e in $\Omega$.

Let $\tilde{G}^{\rho}$ be the approximate Green function of $L_{0}$ on a ball $B$ with pole $x \in B$, and $G$ be the corresponding Green function. In [3], it was shown that for an appropriate subsequence, $\tilde{G}^{\rho_{k}}(y) \rightarrow G(x, y)$ pointwise a.e. on $B$ for a.e. $x \in \frac{1}{2} B$. If $B \subset B^{*}$, then the weak maximum principle, Lemma 2.3 shows that $\tilde{G}^{\rho} \leq \tilde{G}_{*}^{\rho}$ a.e on $B$ if $\tilde{G}_{*}^{\rho}$ is the approximate Green function of $L_{0}$ on $B^{*}$ with pole $x$. Consequently, the inequality $G \leq G^{*}$ holds a.e. on $\frac{1}{2} B \times \frac{1}{2} B$, where $G^{*}$ is the Green function of $L_{0}$ on $B^{*}$.

We also need Lemma (2.7) of [3] in the following slightly modified form. To accomodate this change, we shall indicate the minor alterations needed in the proof of Lemma (2.7) of [3].

Lemma 2.4. Let $B_{j}:=B_{j}\left(x_{0}\right)$ be balls of radius $r_{j}$ for $j=1,2,3$, with $r_{j}<r_{j+1}$. If $\varphi \in H\left(B_{3}\right)$ and $\tilde{\varphi} \leq m$ a.e. in $B_{2}$, then given any $M>m$, and $r_{1}<r<r_{2}$, there exist $\varphi_{k} \in \operatorname{Lip}\left(\bar{B}_{3}\right)$ such that $\varphi_{k} \rightarrow \varphi$ in $H\left(B_{3}\right)$ and $\varphi_{k} \leq M$ a.e. on $B^{*}\left(x_{0}\right)$, a ball of radius $r$.

Proof. As in [3], we pick $h_{k} \in \operatorname{Lip}\left(\bar{B}_{3}\right)$ with $h_{k} \rightarrow \varphi$ in $H\left(B_{3}\right)$. Thus $h_{k} \rightarrow \tilde{\varphi}$ in $L_{v}^{2}\left(B_{3}\right)$, and by using a subsequence, we may assume that $h_{k} \rightarrow \tilde{\varphi}$ a.e. on $B_{3}$. By hypothesis, $\tilde{\varphi} \leq m$ a.e. on $B_{2}$. By Egorov's theorem, given $M>m$, and $\delta>0$, there exist $E \subset B_{2}$, and $k_{0}$ such that $\left|B_{2} \backslash E\right|<\delta$ and $h_{k} \leq M$ on $E$, if $k \geq k_{0}$. Let $\chi \in C_{c}^{\infty}\left(B_{2}\right), 0 \leq \chi \leq 1$, and $\chi \equiv 1$ on $B^{*}$, where $B^{*}:=B^{*}\left(x_{0}\right)$ is a ball of radius $r$. We now define $\varphi_{k}:=h_{k} \chi \wedge M+h_{k}(1-\chi)$. Clearly $\varphi_{k} \in \operatorname{Lip}\left(\bar{B}_{3}\right)$, and $\varphi_{k} \leq M$ a.e. on $B^{*}$. It now remains to show that $\varphi_{k} \rightarrow \varphi$ in $H\left(B_{3}\right)$. Noting that $\varphi_{k}-h_{k}$ is supported on $B_{2}$, the rest of the proof proceeds in the same way as that of Lemma (2.7) of [3].

Remark 2.3. If $\varphi \geq 0$ in the sense of $H\left(B_{3}\right)$, then the $\varphi_{k}$ in Lemma 2.4 can be taken to be non-negative, as can be seen from the definition of $\varphi_{k}$ in the proof.

Remark 2.4. Let $0 \leq m$, and $\varphi \in H_{0}(B)$ such that $\tilde{\varphi} \leq m$ a.e. on $B$. Then given $M>m$, we can choose $\varphi_{k} \in \operatorname{Lip}_{0}(B)$ such that $\varphi_{k} \rightarrow \varphi$ in $H_{0}(B)$, and $\varphi_{k} \leq M$ a.e. on $B$. This follows from the proof of Lemma 2.4 by extending $\tilde{\varphi}$ to be zero outside $B$.

\section{Approximate Green functions.}

The following embedding lemma is useful in the subsequent development. In proving the Lemma, we adapt a method used in [6], in the case of equal weights. 
Lemma 3.1. If $f \in K$, and $B \subset \subset B_{0}$ is a ball of radius $r$, then for any $u \in H_{0}(B)$ the following holds.

$$
\int_{B}|f| \tilde{u}^{2} d x \lesssim \eta(f)(3 r) \int_{B}\langle A \nabla \tilde{u}, \nabla \tilde{u}\rangle .
$$

Proof. Let $G^{*}$ and $G$ be the Green functions of $L_{0}$ on $2 B^{*}$ and $2 B_{0}$, respectively, where $B^{*}$ is a concentric slight enlargement of $B$. As pointed out in the remark following Lemma 2.3, we first observe that $G^{*} \leq G$ a.e. on $B^{*} \times B^{*}$. Let $f_{k}=|f| \wedge(k v), \quad k=1,2, \ldots$, and note that $f_{k} v^{-1} \in L_{v}^{t}(2 B)$ for any $t$. Since $\omega \in A_{2}$, and $\omega \leq v$, we see that $v$ can not vanish on a set of positive Lebesgue measure. Therefore $f_{k} \rightarrow|f|$ a.e. on $B$. Thus once the inequality in the Lemma is shown to hold for $f_{k}$, then by Fatou's Lemma, it will also hold for $f$. So there is no loss of generality in assuming that $f v^{-1} \in L_{v}^{t^{\prime}}(2 B)$ for some $t<\sigma$.

First, let us suppose that $u \in \operatorname{Lip}_{0}(B)$. Let $\zeta:=\left\{\zeta_{k}\right\} \in H_{0}\left(2 B^{*}\right)$ be the Lax-Milgram solution of $L_{0} \zeta=|f| \chi_{B}$ in $2 B^{*}$ and $\zeta=0$ on $\partial\left(2 B^{*}\right)$. Let $\tilde{\zeta}$ be the associated function. Then by the representation theorem in Lemma 2.2, we know that for a.e. $x \in B^{*}$

$$
\begin{aligned}
\tilde{\zeta}(x) & =\int_{B} G^{*}(x, y)|f(y)| d y \leq \int_{B} G(x, y)|f(y)| d y \\
& \leq \int_{3 B_{r}(x) \cap 2 B_{0}} G(x, y)|f(y)| d y .
\end{aligned}
$$

Therefore, $\tilde{\zeta}(x) \leq \eta(f)(3 r)$ for a.e $x \in B^{*}$. By Lemma 2.1, and Lemma 2.4 we pick a sequence $\zeta_{k} \in \operatorname{Lip}_{0}\left(2 B^{*}\right)$ such that $0 \leq \zeta_{k} \rightarrow \zeta$ in $H_{0}\left(2 B^{*}\right)$ and $\zeta_{k} \lesssim \eta(f)(3 r)$ a.e. on $B$. By extending $u$ to be zero outside $B$, we consider the element $\varphi=\left\{u^{2}\right\} \in H_{0}\left(2 B^{*}\right)$. Then, we write

$$
\delta_{k}+\int_{2 B^{*}}|f| u^{2}=\int_{2 B^{*}}\left\langle A \nabla \zeta_{k}, \nabla u^{2}\right\rangle
$$

where

$$
\delta_{k}=\int_{2 B^{*}}\left\langle A \nabla \zeta_{k}, \nabla u^{2}\right\rangle-\int_{2 B^{*}}|f| u^{2} .
$$

By Cauchy-Schwarz inequality, we have

$$
\left\langle A \nabla \zeta_{k}, \nabla u^{2}\right\rangle=2\left\langle A\left(u \nabla \zeta_{k}\right), \nabla u\right\rangle \leq 4 \eta\langle A \nabla u, \nabla u\rangle+\frac{1}{4 \eta}\left\langle A\left(u \nabla \zeta_{k}\right), u \nabla \zeta_{k}\right\rangle,
$$

where $\eta:=\eta(f)(3 r)$. But

$$
\begin{aligned}
& \left\langle A\left(u \nabla \zeta_{k}\right), u \nabla \zeta_{k}\right\rangle \\
& =\left\langle A \nabla \zeta_{k}, \nabla \zeta_{k}\right\rangle u^{2}=\left\langle A \nabla \zeta_{k}, \nabla\left(u^{2} \zeta_{k}\right)\right\rangle-2\left\langle A\left(u \nabla \zeta_{k}\right), \zeta_{k} \nabla u\right\rangle \\
& \leq\left\langle A \nabla \zeta_{k}, \nabla\left(u^{2} \zeta_{k}\right)\right\rangle+\frac{1}{2}\left\langle A\left(u \nabla \zeta_{k}\right), u \nabla \zeta_{k}\right\rangle+2\left\langle A\left(\zeta_{k} \nabla u\right), \zeta_{k} \nabla u\right\rangle .
\end{aligned}
$$


That is,

$$
\left\langle A\left(u \nabla \zeta_{k}\right), u \nabla \zeta_{k}\right\rangle \leq 2\left\langle A \nabla \zeta_{k}, \nabla\left(u^{2} \zeta_{k}\right)\right\rangle+4\langle A \nabla u, \nabla u\rangle \zeta_{k}^{2}
$$

Using (3.3) in (3.2), we obtain

$$
\begin{aligned}
& \int_{2 B^{*}}\left\langle A \nabla \zeta_{k}, \nabla u^{2}\right\rangle \\
& \leq 4 \eta \int_{2 B^{*}}\langle A \nabla u, \nabla u\rangle+\frac{1}{2 \eta} \int_{2 B^{*}}\left\langle A \nabla \zeta_{k}, \nabla\left(u^{2} \zeta_{k}\right)\right\rangle \\
& \quad+\frac{1}{\eta} \int_{2 B^{*}}\langle A \nabla u, \nabla u\rangle \zeta_{k}^{2} \\
& =4 \eta \int_{2 B^{*}}\langle A \nabla u, \nabla u\rangle+\frac{1}{2 \eta} \int_{2 B^{*}}|f| u^{2} \zeta_{k}+\frac{1}{\eta} \int_{2 B^{*}}\langle A \nabla u, \nabla u\rangle \zeta_{k}^{2}+\frac{1}{2 \eta} \gamma_{k},
\end{aligned}
$$

where

$$
\gamma_{k}:=\int_{2 B^{*}}\left\langle A \nabla \zeta_{k}, \nabla\left(u^{2} \zeta_{k}\right)\right\rangle-\int_{2 B^{*}}|f| u^{2} \zeta_{k} .
$$

Using this in (3.1), and recalling that $\operatorname{supp}(u) \subset B$, and $0 \leq \zeta_{k} \lesssim \eta$ a.e. on $B$, we obtain

$$
\delta_{k}+\int_{B}|f| u^{2} \lesssim 5 \eta \int_{B}\langle A \nabla u, \nabla u\rangle+\frac{1}{2} \int_{B}|f| u^{2}+\frac{1}{2 \eta} \gamma_{k} .
$$

By $(2.2),\left\{\varphi_{k}\right\}:=\left\{u^{2} \zeta_{k}\right\}$ is easily seen to be bounded in $H_{0}\left(2 B^{*}\right)$. Therefore there is a weakly convergent subsequence which we continue to denote by $\left\{\varphi_{k}\right\}$. Using this subsequence, and recalling that $\zeta \in H_{0}\left(2 B^{*}\right)$ is the Lax-Milgram solution of $L_{0} \zeta=|f| \chi_{B}$ in $2 B^{*}$ and $\zeta=0$ on $\partial\left(2 B^{*}\right)$, we see by Remark 2.2 that $\delta_{k} \rightarrow 0$, and $\gamma_{k} \rightarrow 0$ as $k \rightarrow \infty$. Therefore, taking the limit as $k \rightarrow \infty$ in the inequality (3.4), we conclude

$$
\int_{B}|f| u^{2} \lesssim 5 \eta \int_{B}\langle A \nabla u, \nabla u\rangle+\frac{1}{2} \int_{B}|f| u^{2},
$$

from which follows the desired result when $u \in \operatorname{Lip}_{0}(B)$.

To prove the Lemma for $u \in H_{0}(B)$, suppose $u=\left\{u_{k}\right\}, u_{k} \in \operatorname{Lip}_{0}(B)$. For each $k$, we have

$$
\int_{B}|f| u_{k}^{2} \lesssim 10 \eta \int_{B}\left\langle A \nabla u_{k}, \nabla u_{k}\right\rangle
$$

Take a subsequence of $\left\{u_{k}\right\}$ that converges pointwise a.e. to $\tilde{u}$ on $B$. By appealing to (2.3), and Fatou's Lemma we get the desired result after taking the limit as $k \rightarrow \infty$.

Remark 3.1. Let $f \in K$, and $B \subset \subset B_{0}$ be a ball. Using Hölder inequality, followed by an application of Lemma 3.1, the map

$$
\varphi \mapsto \int_{B} f \tilde{\varphi}
$$


is seen to be continuous on $H_{0}(B)$. Therefore, by the Lax-Milgram theorem there is a unique $\zeta \in H_{0}(B)$ such that

$$
a_{0}(\zeta, \varphi)=\int_{B} f \tilde{\varphi}, \quad \text { for } \varphi \in H_{0}(B) .
$$

We will also refer to $\zeta$ as the Lax-Milgram solution of $L_{0} \zeta=f$ on $B, \zeta=$ 0 on $\partial B$.

Lemma 3.2. Let $f \in K$, and $B \subset B_{0}$ be a ball of radius $r$.

(1) If $\tilde{G}^{\rho}$ is the approximate Green function of $L_{0}$ on $B$, then

$$
\int_{B}|f| \tilde{G}^{\rho} \lesssim \eta(f)(2 r)
$$

(2) If $\xi \in H_{0}(B)$ is the Lax-Milgram solution of $L_{0} \xi=|f|$ in $B$, then

$$
\tilde{\xi}(x) \lesssim \eta(f)(2 r), \quad \text { for a.e } \quad x \in B .
$$

Proof. First we show that if $\zeta \in H_{0}(2 B)$ is the Lax-Milgram solution of $L_{0} \zeta=|f| \chi_{B}$ in $2 B$, then $\tilde{\zeta}(x) \lesssim \eta(f)(2 r)$ for a.e. $x \in 2 B$. To this end, let us write $|f|^{(k)}:=|f| \wedge(k v), \quad k=1,2,3 \cdots$, and $|f|^{(0)}:=|f|$. As in the proof of Lemma 3.1, we can argue that $|f|^{(k)} \rightarrow|f|$ pointwise a.e. on $B$. By (2.1) and Lemma 3.1 the map

$$
\varphi \in H_{0}(2 B) \mapsto \int_{B^{*}}|f|^{(k)} \tilde{\varphi},
$$

is seen to be continuous on $H_{0}(2 B)$ for all $k=0,1,2, \cdots$. Let $\zeta^{(k)} \in H_{0}(2 B)$ be the Lax-Milgram solution of $L_{0} \zeta=|f|^{(k)} \chi_{B^{*}}$ on $2 B$. Here $B^{*}$ is a ball concentric to $B$ but with radius $(1-\epsilon) r$ for small $\epsilon>0$. For $k=1,2,3, \cdots$, note that $|f|^{(k)} v^{-1} \in L_{v}^{t^{\prime}}(B), t<\sigma$. Then, by the representation formula of Lemma 2.2, we have for a.e. $x \in B$, and $k=1,2, \cdots$,

$$
\begin{aligned}
\tilde{\zeta}^{(k)}(x) & =\int_{B^{*}} G_{r}(x, y)|f|^{(k)}(y) d y \leq \int_{B^{*}} G(x, y)|f|^{(k)}(y) d y \\
& \leq \int_{2 B_{r}(x) \cap 2 B_{0}} G(x, y)|f(y)| d y \leq \eta(f)(2 r),
\end{aligned}
$$

where $G_{r}$ and $G$ denote the Green functions of $L_{0}$ on $2 B$ and $2 B_{0}$ respectively. We have used the fact that $G_{r} \leq G$ on $B$, which is valid by the weak maximum principle, Lemma 2.3. By Lemma 2.4, there is a sequence $\left\{\zeta_{m}^{(k)}\right\}$ in $\operatorname{Lip}_{0}(2 B)$ such that $\zeta_{m}^{(k)} \rightarrow \zeta^{(k)}$ in $H_{0}(2 B)$, and $\zeta_{m}^{(k)} \lesssim \eta(f)(2 r)$ a.e. on a ball concentric with $B$, and of radius strictly between that of $B^{*}$, and $B$. Now let us observe that $\zeta^{(k)}$, for $k=1,2,3, \cdots$, is the weak solution of $L_{0} \zeta=0$ on $2 B \backslash B^{*}$ such that $C \eta(f)(2 r)-\zeta_{m}^{(k)} \geq 0$ on a neighborhood of $\partial\left(2 B \backslash B^{*}\right)$. Therefore by the weak maximum principle, Lemma 2.3, we conclude that $\tilde{\zeta}^{(k)} \lesssim \eta(f)(2 r)$ on $2 B \backslash B^{*}$, and hence on $2 B$. To show 
that the same bound holds for $\zeta:=\zeta^{(0)}$, let $\tilde{G}_{*}^{\rho}$ be the approximate Green function of $L_{0}$ on $2 B$, and let us observe that

$f_{B_{\rho}}\left(\tilde{\zeta}-\tilde{\zeta}^{(k)}\right) v=a_{0}\left(G_{*}^{\rho}, \zeta-\zeta^{(k)}\right)=a_{0}\left(\zeta-\zeta^{(k)}, G_{*}^{\rho}\right)=\int_{B}\left(|f|-|f|^{(k)} \chi_{B^{*}}\right) \tilde{G}_{*}^{\rho}$,

for all $k$. Since $|f| \tilde{G}_{*}^{\rho} \in L^{1}(B)$, we invoke the Lebesgue dominated convergence theorem to conclude that

$$
\begin{aligned}
f_{B_{\rho}} \tilde{\zeta} v & =\lim _{k \rightarrow \infty} f_{B_{\rho}} \tilde{\zeta}^{(k)} v+\int_{B}|f|\left(1-\chi_{B^{*}}\right) \tilde{G}_{*}^{\rho} \\
& \leq C \eta(f)(2 r)+\int_{B \backslash B^{*}}|f| \tilde{G}_{*}^{\rho} .
\end{aligned}
$$

After letting $\epsilon \rightarrow 0$, we obtain

$$
f_{B_{\rho}} \tilde{\zeta} v \lesssim \eta(f)(2 r)
$$

This leads to the claimed estimate after taking the limit as $\rho \rightarrow 0$, namely we get,

$$
\tilde{\zeta}(x) \lesssim \eta(f)(2 r), \quad \text { for a.e. } \quad x \in 2 B .
$$

We now use this result to prove the statement in (1). To see this, let us take the Lax-Milgram solution $\zeta$ of $L_{0} \zeta=|f| \chi_{B}$ in $H_{0}(2 B)$. Let $\tilde{G}^{\rho}$, and $\tilde{G}_{*}^{\rho}$ be the approximate Green functions of $L_{0}$ on $B$, and $2 B$ respectively. Since $G_{*}^{\rho}-G^{\rho}$ is a solution of $L_{0}$ in $B$, and $\tilde{G}_{*}^{\rho}-\tilde{G}^{\rho} \geq 0$ near $\partial B$, by Lemma 2.3 we note that $\tilde{G}^{\rho} \leq \tilde{G}_{*}^{\rho}$ on $B$. Therefore, by $(3.5)$ above,

$$
\int_{B}|f| \tilde{G}^{\rho} \leq \int_{B}|f| \tilde{G}_{*}^{\rho}=a_{0}\left(\zeta, G_{*}^{\rho}\right)=a_{0}\left(G_{*}^{\rho}, \zeta\right)=f_{B_{\rho}} \tilde{\zeta} v \lesssim \eta(f)(2 r) .
$$

The statement in (2) is now an easy consequence of (1). To see this, let $\xi \in H_{0}(B)$ be the Lax-Milgram solution of $L_{0} \xi=|f|$ in $B$. Let $\tilde{G}^{\rho}$ be the approximate Green function of $L_{0}$ on $B$. Then

$$
f_{B_{\rho}} \tilde{\xi} v=a_{0}\left(G^{\rho}, \xi\right)=a_{0}\left(\xi, G^{\rho}\right)=\int_{B}|f| \tilde{G}^{\rho} \lesssim \eta(2 r) .
$$

Taking the limit as $\rho \rightarrow 0$, we obtain the desired result.

The next Lemma is a slight extension of (2.3), and we will use it repeatedly.

Lemma 3.3. Let $u=\left\{u_{k}\right\}, \varphi=\left\{\varphi_{k}\right\}$ be in $H(B)$. If $\left\{\zeta_{k}\right\}$ is a bounded sequence in $L^{\infty}(B)$ that converges pointwise a.e. to $\zeta \in L^{\infty}(B)$, then

$$
\int_{B}\left\langle A \nabla u_{k}, \nabla \varphi_{k}\right\rangle \zeta_{k} \rightarrow \int_{B}\langle A \nabla \tilde{u}, \nabla \tilde{\varphi}\rangle \zeta, \quad \text { as } \quad k \rightarrow \infty .
$$


Proof. Since

$$
\int_{B}\left|\left\langle A \nabla u_{k}, \nabla \varphi_{k}\right\rangle \zeta_{k}-\langle A \nabla \tilde{u}, \nabla \tilde{\varphi}\rangle \zeta\right|
$$

is not bigger than

$$
\left\|\zeta_{k}\right\|_{\infty} \int\left|\left\langle A \nabla u_{k}, \nabla \varphi_{k}\right\rangle-\langle A \nabla \tilde{u}, \nabla \tilde{\varphi}\rangle\right|+\int\left|\langle A \nabla \tilde{u}, \nabla \tilde{\varphi}\rangle \zeta \| \zeta_{k}-\zeta\right|,
$$

and $\langle A \nabla \tilde{u}, \nabla \tilde{\varphi}\rangle \zeta \in L^{1}(B)$ the Lemma follows from (2.3), and the Lebesgue dominated convergence theorem.

Let us now consider the general elliptic operator:

$$
M u:=-\operatorname{div}(A(x) \nabla u+\mathbf{c}(x) u)+\mathbf{b}(x) \cdot \nabla u+V(x) u,
$$

where, in addition to (1.5) we also assume that $|\mathbf{c}|^{2} \omega^{-1} \in K$. With $M$, and its adjoint operator

$$
M^{*} u:=-\operatorname{div}(A(x) \nabla u+\mathbf{b}(x) u)+\mathbf{c}(x) \cdot \nabla u+V(x) u,
$$

we associate the bilinear forms $D(.,$.$) and D_{*}(.,$.$) as follows. Fix a ball$ $B \subset \subset B_{0}$ of radius $r$, and let

$$
D(u, \varphi):=\int_{B}\langle A \nabla u, \nabla \varphi\rangle+\mathbf{c}(x) \cdot(\nabla \varphi) u+\mathbf{b}(x) \cdot \nabla u \varphi+V u \varphi,
$$

and $D_{*}(u, \varphi):=D(\varphi, u)$, for all $u, \varphi \in \operatorname{Lip}_{0}(B)$. Observe that by Hölder inequality and Lemma 3.1, it follows

$$
\left|D(u, \varphi)-a_{0}(u, \varphi)\right| \lesssim \vartheta(r)\|u\|_{0}\|\varphi\|_{0}, \quad u, \varphi \in \operatorname{Lip}_{0}(B),
$$

where $\vartheta(r):=\left(\eta\left(|\mathbf{c}|^{2} \omega^{-1}\right)(3 r)\right)^{1 / 2}+\left(\eta\left(|\mathbf{b}|^{2} \omega^{-1}\right)(3 r)\right)^{1 / 2}+\eta(V)(3 r)$. Therefore, we get

$$
|D(u, \varphi)| \lesssim(1+\vartheta(r))\|u\|_{0}\|\varphi\|_{0}, \quad u, \varphi \in \operatorname{Lip}_{0}(B) .
$$

Thus if $u=\left\{u_{k}\right\}, \varphi=\left\{\varphi_{k}\right\}, u_{k}, \varphi_{k} \in \operatorname{Lip}_{0}(B)$ are elements of $H_{0}(B)$ then the above inequality shows that $\left\{D\left(u_{k}, \varphi_{k}\right)\right\}$ is a Cauchy sequence and hence $\lim _{k} D\left(u_{k}, \varphi_{k}\right)$ exists. Therefore we define

$$
D(u, \varphi):=\lim _{k} D\left(u_{k}, \varphi_{k}\right) .
$$

Having defined $D(u, \varphi)$ for $u, \varphi \in H_{0}(B)$, the inequality (3.7) still holds for any $u, \varphi \in H_{0}(B)$. As a result of this inequality we see that for a fixed $u \in H_{0}(B)$, the map $\varphi \mapsto D(u, \varphi)$ is a continuous linear functional on $H_{0}(B)$.

Using (3.6) one also obtains $a_{0}(u, u)(1-C \vartheta(r)) \lesssim D(u, u)$, for $u \in$ $\operatorname{Lip}_{0}(B)$. Therefore for sufficiently small $r_{0}$, and all $0<r \leq r_{0}$, we have

$$
\|u\|_{0}^{2} \lesssim D(u, u), \quad u \in H_{0}(B)
$$

so that $D(.,$.$) is a coercive bilinear form on H_{0}(B)$. 
Given $f \in K$, we shall say that $u \in H_{0}(B)$ is a weak solution of $M u=f$ in $B$ if

$$
D(u, \varphi)=\int_{B} f \tilde{\varphi}
$$

for all $\varphi \in H_{0}(B)$. Similar statements and definitions hold for the adjoint operator $M^{*}$ and the associated bilinear form $D_{*}(.,$.$) .$

The following remark will be useful at several stages in our subsequent proofs.

Remark 3.2. Let $f \in K$, and $u=\left\{u_{k}\right\} \in H_{0}(B)$ be a weak solution of $M u=f$ in $B$. If $\left\{v_{k}\right\}$ is a bounded, weakly convergent sequence in $H_{0}(B)$, then

$$
\lim _{k \rightarrow \infty}\left[D\left(u_{k}, v_{k}\right)-\int_{B} f \tilde{v}_{k}\right]=0 .
$$

This can be verified along the lines of argument given in Remark 2.2, since the linear functionals

$$
\varphi \mapsto D(u, \varphi), \quad \text { and } \quad \varphi \mapsto \int_{B} f \tilde{\varphi}
$$

are continuous on $H_{0}(B)$.

Lemma 3.4. Suppose $f v^{-1} \in L_{v}^{p}(B)$ for some $p>\frac{\sigma}{\sigma-1}$, and some $B \subset B_{0}$, where $B$ is a ball of raduis $r$. Then there is a unique solution $u$ of $L_{0} u=f$ in $H_{0}(B)$, and the following estimate holds.

$$
\|\tilde{u}\|_{L^{\infty}(B)} \leq C r^{2} \frac{v(B)^{\frac{1}{p^{\prime}}}}{\omega(B)}\left\|f v^{-1}\right\|_{L_{v}^{p}(B)} .
$$

Proof. The existence and uniqueness follows by the Lax-Milgram Theorem as pointed out in Remark 2.1.

Let $\tilde{G}^{\rho}$ be the approximate Green function of $L_{0}$ on $B$ with pole $y \in B$. Then

$$
f_{B_{\rho}} \tilde{u} v=a_{0}\left(G^{\rho}, u\right)=a_{0}\left(u, G^{\rho}\right)=\int_{B} f \tilde{G}^{\rho},
$$

so that by Hölder's inequality,

$$
\begin{aligned}
\left|f_{B_{\rho}} \tilde{u} v\right|=\left|\int_{B} f \tilde{G}^{\rho}\right| & \leq\left\|f v^{-1}\right\|_{L_{v}^{p}(B)}\left(\int_{B}\left(\tilde{G}^{\rho}\right)^{p^{\prime}} v\right)^{\frac{1}{p^{\prime}}} \\
& \leq C r^{2} \frac{v(B)^{\frac{1}{p^{\prime}}}}{\omega(B)}\left\|f v^{-1}\right\|_{L_{v}^{p}(B)} .
\end{aligned}
$$

In the last inequality, we used, (see [3]) the fact that, when $1<p^{\prime}<\sigma$,

$$
\left(\int_{B}\left(\tilde{G}^{\rho}\right)^{p^{\prime}} v\right)^{\frac{1}{p^{\prime}}} \leq C r^{2} \frac{v(B)^{\frac{1}{p^{\prime}}}}{\omega(B)},
$$


where $C$ is independent of $\rho$ and the pole of $\tilde{G}^{\rho}$. If we now let $\rho \rightarrow 0$, we conclude

$$
|\tilde{u}(y)| \leq C r^{2} \frac{v(B)^{\frac{1}{p^{\prime}}}}{\omega(B)}\left\|f v^{-1}\right\|_{L_{v}^{p}(B)} .
$$

This and the arbitrariness of $y \in B$ establishes the Lemma.

Remark 3.3. Let $G^{\rho}$ be the approximate Green function of $L_{0}$ on $B$. Thus $G^{\rho}$ is the Lax-Milgram solution of $L_{0} G^{\rho}=v \chi_{B_{\rho}}\left[v\left(B_{\rho}\right)\right]^{-1}$. Therefore, by Lemma 3.4, $\left\|\tilde{G}^{\rho}\right\|_{L^{\infty}(B)} \leq C$ for some constant $C$ depending on $\rho$, the pole of $G^{\rho}$, and $v$.

For the next Lemma, given $B \subset B_{0}$ we take $f \in L^{1}(B)$ such that the map

$$
\varphi \mapsto \int_{B} f \tilde{\varphi}, \quad \varphi \in H_{0}(B)
$$

is continuous on $H_{0}(B)$. Furthermore, we require that

$$
\int_{B}|f| \tilde{G}^{\rho}=O(1), \quad \text { as } \quad \rho \rightarrow 0^{+},
$$

where $\tilde{G}^{\rho}$ is the approximate Green function of $L_{0}$ on $B$.

Lemma 3.5. If $u \in H_{0}(B)$ is the unique solution of $L_{0} u=f$ on $B$, then $u$ has a representative $u=\left\{u_{k}\right\}, u_{k} \in \operatorname{Lip}_{0}(B)$ such that $\left\|u_{k}\right\|_{\infty} \leq M$ uniformly in $k$ for some constant $M$.

Proof. Let $u^{(+)} \in H_{0}(B)$, and $u^{(-)} \in H_{0}(B)$ be the solutions of $L_{0} u^{(+)}=$ $f^{+}$, and $L_{0} u^{(-)}=f^{-}$respectively. Here $f^{+}:=\max \{0, f\}$, and $f^{-}:=$ $\max \{0,-f\}$. If $\tilde{u}^{(+)}$, and $\tilde{u}^{(-)}$are the associated functions, then

$$
f_{B_{\rho}} \tilde{u}^{( \pm)} v=a_{0}\left(G^{\rho}, u^{( \pm)}\right)=a_{0}\left(u^{( \pm)}, G^{\rho}\right)=\int_{B} f^{ \pm} \tilde{G}^{\rho} \leq C .
$$

Taking the limit as $\rho \rightarrow 0$, we conclude that $\tilde{u}^{( \pm)} \leq C$ a.e. on $B$. By Lemma 2.4 (see Remark 2.4), the solutions $u^{(+)}$, and $u^{(-)}$have representatives $u^{(+)}=\left\{u_{k}^{(+)}\right\}, u_{k}^{(+)} \in \operatorname{Lip}_{0}(B)$, and $u^{(-)}=\left\{u_{k}^{(-)}\right\}, u_{k}^{(-)} \in \operatorname{Lip}_{0}(B)$ such that

$$
u_{k}^{(+)} \lesssim C, \quad \text { and } \quad u_{k}^{(-)} \lesssim C
$$

a.e. on $B$. By Lemma 2.1, we can in fact choose such representatives to satisfy

$$
0 \leq u_{k}^{(+)} \lesssim C, \quad \text { and } \quad 0 \leq u_{k}^{(-)} \lesssim C
$$

a.e. on $B$. Now let $u^{*}=\left\{u_{k}^{(+)}-u_{k}^{(-)}\right\}$. It is easy to verify that $u^{*} \in H_{0}(B)$ is a solution of $L_{0} u=f$ on $B$. By uniqueness, we must then have $u=$ $\left\{u_{k}^{(+)}-u_{k}^{(-)}\right\}$, and this representation satisfies the condition stated in the Lemma. 
From now on, we will assume that $\mathbf{c} \equiv 0$ in the bilinear forms $D(.,$.$) , and$ $D_{*}(.,$.$) .$

Theorem 3.1. Let $B \subset \subset B_{0}$ be a ball of radius $r$, and let $f v^{-1} \in L_{v}^{p}(B)$ for some $p>\frac{\sigma}{\sigma-1}$. Then there is $r_{0}$ such that if $0<r \leq r_{0}$, there is a unique solution $u \in H_{0}(B)$ of $L u=f$ in $B$ and it satisfies the estimate

$$
\|\tilde{u}\|_{L^{\infty}(B)} \leq C r^{2} \frac{v(B)^{\frac{1}{p^{\prime}}}}{\omega(B)}\left\|f v^{-1}\right\|_{L_{v}^{p}(B)},
$$

for some constant $C$.

Proof. Choose $r_{0}$ such that the bounded bilinear form $D(.,$.$) is coercive on$ $H_{0}(B)$, whenever $B$ is a ball of radius $r$, with $0<r \leq r_{0}$. Since $\varphi \rightarrow \int_{B} f \tilde{\varphi}$ is a continuous linear functional on $H_{0}(B)$, by the Lax-Milgram theorem there is a unique $u \in H_{0}(B)$ such that

$$
D(u, \varphi)=\int_{B} f \tilde{\varphi}, \quad \varphi \in H_{0}(B) ;
$$

that is $L u=f$ in $B$. Moreover, by Hölder's and Sobolev inequality,

$$
\|u\|_{0} \leq C\left\|f v^{-1}\right\|_{L_{v}^{p}(B)} .
$$

We want to show that for some constant $C$,

$$
\|\tilde{u}\|_{L^{\infty}(B)} \leq C r^{2} \frac{v(B)^{\frac{1}{p^{\prime}}}}{\omega(B)}\left\|f v^{-1}\right\|_{L_{v}^{p}(B)} .
$$

Let $u_{-1} \equiv 0$, and we inductively define $u_{j} \in H_{0}(B), j=0,1,2, \cdots$, as the unique element for which

$$
a_{0}\left(u_{j}, \varphi\right)=\int_{B}\left(f-\mathbf{b} \cdot \nabla \tilde{u}_{j-1}-V \tilde{u}_{j-1}\right) \tilde{\varphi}, \quad \text { for all } \quad \varphi \in H_{0}(B),
$$

so that $u_{j}$ is the solution of $L_{0} u+\mathbf{b} \cdot \nabla \tilde{u}_{j-1}+V \tilde{u}_{j-1}=f$ in $H_{0}(B)$. This is possible, since for a given $u_{j-1} \in H_{0}(B)$, the map

$$
\varphi \mapsto \int_{B}\left(f-\mathbf{b} \cdot \nabla \tilde{u}_{j-1}-V \tilde{u}_{j-1}\right) \tilde{\varphi}
$$

is a continuous linear functional on $H_{0}(B)$. Suppose that $\tilde{G}^{\rho}$ is the approximate Green function of $L_{0}$ on $B$. We now claim that for each $j=0,1,2, \cdots$, we can choose a representative $u_{j}=\left\{u_{j}^{(k)}\right\}, u_{j}^{(k)} \in \operatorname{Lip}_{0}(B)$, such that

$$
\left\|u_{j}^{(k)}\right\|_{\infty} \leq M_{j}, \quad \text { and } \quad \int_{B}\left|\nabla \tilde{u}_{j}\right|^{2} \tilde{G}^{\rho} \omega=O(1), \quad \text { as } \quad \rho \rightarrow 0^{+},
$$

for some positive constant $M_{j}$ independent of $k$. We show this by induction on $j$. Since $\tilde{G}^{\rho}$ is essentially bounded, and since $G^{\rho} \geq 0$, by Lemma 2.4 (or see Remark 2.4) we can take a representative $G^{\rho}=\left\{G_{k}^{\rho}\right\}, G_{k}^{\rho} \in \operatorname{Lip}_{0}(B)$ such that $0 \leq G_{k}^{\rho} \leq C$ a.e. on B for some constant $C$ independent of $k$. 
Since $u_{0} \in H_{0}(B)$ is the solution of $L_{0} u=f$, by Lemma 3.5 we can choose a representative $u_{0}=\left\{u_{0}^{(k)}\right\}, u_{0}^{(k)} \in \operatorname{Lip}_{0}(B)$ such that $u_{0}^{(k)}$ is uniformly bounded on $B$. Consequently, one can use (2.2) to show that $\left\{u_{0}^{(k)} G_{k}^{\rho}\right\}$, and $\left\{\left(u_{0}^{(k)}\right)^{2}\right\}$ are bounded in $H_{0}(B)$. Then for some subsequences, $\varphi_{0}^{(k)}:=$ $u_{0}^{(k)} G_{k}^{\rho}$, and $\psi_{0}^{(k)}:=\left(u_{0}^{(k)}\right)^{2}$ are weakly convergent in $H_{0}(B)$. Using a further subsequence if necessary, we can assume that $\varphi_{0}^{(k)} \rightarrow \tilde{u}_{0} \tilde{G}^{\rho}$ a.e. on $B$. Let us now observe that

$$
\begin{aligned}
& \int_{B}\left\langle A \nabla u_{0}^{(k)}, \nabla u_{0}^{(k)}\right\rangle G_{k}^{\rho} \\
& =\int_{B}\left\langle A \nabla u_{0}^{(k)}, \nabla\left(u_{0}^{(k)} G_{k}^{\rho}\right)\right\rangle-\frac{1}{2} \int_{B}\left\langle A \nabla G_{k}^{\rho}, \nabla\left(u_{0}^{(k)}\right)^{2}\right\rangle \\
& =\delta_{k}+\int_{B} f u_{0}^{(k)} G_{k}^{\rho}-\frac{1}{2} f_{B_{\rho}}\left(u_{0}^{(k)}\right)^{2} v \\
& \leq \delta_{k}+\int_{B}|f| u_{0}^{(k)} G_{k}^{\rho},
\end{aligned}
$$

where

$$
\begin{aligned}
\delta_{k}:= & \int\left\langle A \nabla u_{0}^{(k)}, \nabla\left(u_{0}^{(k)} G_{k}^{\rho}\right)\right\rangle-\frac{1}{2} \int\left\langle A \nabla G_{k}^{\rho}, \nabla\left(u_{0}^{(k)}\right)^{2}\right\rangle \\
& -\int f u_{0}^{(k)} G_{k}^{\rho}+\frac{1}{2} f_{B_{\rho}}\left(u_{0}^{(k)}\right)^{2} v,
\end{aligned}
$$

and the first three integrals are over $B$. We now take the limit as $k \rightarrow \infty$. By Remark 2.2, we observe that $\delta_{k} \rightarrow 0$. Then by Lemma 3.3, Lebesgue dominated convergence theorem, and the fact that $\delta_{k} \rightarrow 0$, we obtain

$$
\int_{B}\left\langle A \nabla \tilde{u}_{0}, \nabla \tilde{u}_{0}\right\rangle \tilde{G}^{\rho} \leq \int_{B}|f| \tilde{u}_{0} \tilde{G}^{\rho} .
$$

Using (1-1), this leads to the estimate

$$
\begin{aligned}
\int_{B}\left|\nabla \tilde{u}_{0}\right|^{2} \tilde{G}^{\rho} \omega & \leq\left\|\tilde{u}_{0}\right\|_{\infty} \int_{B}|f| \tilde{G}^{\rho} \leq C\left\|\tilde{u}_{0}\right\|_{\infty} r^{2} \frac{v(B)^{\frac{1}{p^{\prime}}}}{\omega(B)}\left\|f v^{-1}\right\|_{L_{v}^{p}(B)} \\
& =A_{0}\left\|\tilde{u}_{0}\right\|_{\infty}\left\|f v^{-1}\right\|_{L_{v}^{p}(B)},
\end{aligned}
$$

where we have also used (3.8) in the penultimate inequality and $A_{0}$ stands for the expression $C r^{2} v(B)^{\frac{1}{p^{\prime}}} / \omega(B)$. This completes the first induction step.

Let us now suppose that $u_{j}$ has a representative $u_{j}=\left\{u_{j}^{(k)}\right\}, u_{j}^{(k)} \in$ $\operatorname{Lip}_{0}(B)$ and that (3.9) holds for the index $j$ and some constant $M_{j}$. Then by Lemma 3.2, Hölder's inequality, and assumption (3.9), we see that

$$
\int_{B}\left|f-\mathbf{b} \cdot \nabla \tilde{u}_{j}-V \tilde{u}_{j}\right| \tilde{G}^{\rho} \leq C
$$


for some constant $C$ independent of $\rho$. Thus by Lemma 3.5, we can find a representative $u_{j+1}=\left\{u_{j+1}^{(k)}\right\}, u_{j+1}^{(k)} \in \operatorname{Lip}_{0}(B)$ such that $\left\|u_{j+1}^{(k)}\right\|_{\infty} \leq M_{j+1}$ on $B$ for some positive constant $M_{j+1}$ independent of $k$. The rest of the argument proceeds in exactly the same way as for the $j=0$ case. This completes the induction, thereby proving the claim that (3.9) holds for all $j$.

Now let $\xi_{j}:=u_{j}-u_{j-1}$, for $j=0,1,2, \cdots$, where we take the representation $\xi_{j}:=\left\{\xi_{j}^{(k)}\right\}$ with $\xi_{j}^{(k)}:=u_{j}^{(k)}-u_{j-1}^{(k)}$. Then the $\xi_{j}$ satisfy

$$
a_{0}\left(\xi_{j}, \varphi\right)=-\int_{B}\left(\mathbf{b} \cdot \nabla \tilde{\xi}_{j-1}+V \tilde{\xi}_{j-1}\right) \tilde{\varphi}
$$

for all $\varphi \in H_{0}(B)$, and $j=1,2, \ldots$.

As a result of (3.9), we have

$$
\left\|\tilde{\xi}_{j}\right\|_{\infty}<\infty, \text { and } \int_{B}\left|\nabla \tilde{\xi}_{j}\right|^{2} \tilde{G}^{\rho} \omega=O(1), \quad \text { as } \rho \rightarrow 0^{+}
$$

For notational convenience, let us introduce the following. For some sufficiently small $\rho_{0}$, and for $j=0,1,2, \ldots$, let

$\vartheta:=\sqrt{\eta\left(|\mathbf{b}|^{2} \omega^{-1}\right)(3 r)}+\eta(V)(3 r), \quad$ and $\quad \tau_{j}:=\sup _{0<\rho \leq \rho_{0}}\left(\int_{B}\left|\nabla \tilde{\xi}_{j}\right|^{2} \tilde{G}^{\rho} \omega\right)^{\frac{1}{2}}$.

Using these notations, and using Lemma 3.2, we find that

$$
\begin{aligned}
f_{B_{\rho}} \tilde{\xi}_{j} v & =a_{0}\left(G^{\rho}, \xi_{j}\right)=a_{0}\left(\xi_{j}, G^{\rho}\right)=-\int_{B}\left(\mathbf{b} \cdot \nabla \tilde{\xi}_{j-1}+V \tilde{\xi}_{j-1}\right) \tilde{G}^{\rho} \\
& \leq\left(\int_{B}|\mathbf{b}|^{2} \omega^{-1} \tilde{G}^{\rho}\right)^{\frac{1}{2}} \cdot\left(\int_{B}\left|\nabla \tilde{\xi}_{j-1}\right|^{2} \tilde{G}^{\rho} \omega\right)^{\frac{1}{2}}+\left\|\tilde{\xi}_{j-1}\right\|_{\infty} \int_{B}|V| \tilde{G}^{\rho} \\
& \leq \vartheta\left(\tau_{j-1}+\left\|\tilde{\xi}_{j-1}\right\|_{\infty}\right)
\end{aligned}
$$

After letting $\rho \rightarrow 0$, we obtain

$$
\left\|\tilde{\xi}_{j}\right\|_{\infty} \leq \vartheta\left(\tau_{j-1}+\left\|\tilde{\xi}_{j-1}\right\|_{\infty}\right)
$$

As a consequence of (2.2), and (3.9) one can see that the sequence $\left\{\xi_{j}^{(k)} G_{k}^{\rho}\right\}$ is bounded in $H_{0}(B)$. Then for an appropriate subsequence, $\varphi_{j}^{(k)}:=\xi_{j}^{(k)} G_{k}^{\rho}$ and $\psi_{j}^{(k)}:=\left(\xi_{j}^{(k)}\right)^{2}$ are weakly convergent in $H_{0}(B)$. Without loss of generality, 
we can assume that $\varphi_{j}^{(k)} \rightarrow \tilde{\xi}_{j} \tilde{G}^{\rho}$ pointwise a.e. on $B$. We now observe that

$$
\begin{aligned}
\int_{B}\left\langle A \nabla \xi_{j}^{(k)}, \nabla \xi_{j}^{(k)}\right\rangle G_{k}^{\rho} & =\int_{B}\left\langle A \nabla \xi_{j}^{(k)}, \nabla\left(\xi_{j}^{(k)} G_{k}^{\rho}\right)\right\rangle-\frac{1}{2} \int_{B}\left\langle A \nabla G_{k}^{\rho}, \nabla\left(\xi_{j}^{(k)}\right)^{2}\right\rangle \\
& =\delta_{j}^{(k)}+\int_{B}\left(\mathbf{b} \cdot \nabla \tilde{\xi}_{j-1}+V \tilde{\xi}_{j-1}\right) \xi_{j}^{(k)} G_{k}^{\rho}-f_{B_{\rho}}\left(\xi_{j}^{(k)}\right)^{2} v \\
& \leq \delta_{j}^{(k)}+\int_{B}\left(\mathbf{b} \cdot \nabla \tilde{\xi}_{j-1}+V \tilde{\xi}_{j-1}\right) \xi_{j}^{(k)} G_{k}^{\rho}
\end{aligned}
$$

where $\delta_{j}^{(k)}$ is given by

$$
\begin{aligned}
\int_{B}\left\langle A \nabla \xi_{j}^{(k)}, \nabla\left(\xi_{j}^{(k)} G_{k}^{\rho}\right)\right\rangle & -\frac{1}{2} \int_{B}\left\langle A \nabla G_{k}^{\rho}, \nabla\left(\xi_{j}^{(k)}\right)^{2}\right\rangle \\
& -\int_{B}\left(\mathbf{b} \cdot \nabla \tilde{\xi}_{j-1}+V \tilde{\xi}_{j-1}\right) \xi_{j}^{(k)} G_{k}^{\rho}+f_{B_{\rho}}\left(\xi_{j}^{(k)}\right)^{2} v .
\end{aligned}
$$

Notice that by Remark $2.2, \delta_{j}^{(k)} \rightarrow 0$ as $k \rightarrow \infty$. Therefore taking the limit in the last inequality, as $k \rightarrow \infty$, applying Lemma 3.3, and the Lebesgue dominated convergence theorem, followed by an application of Hölder inequality and Lemma 3.1, we obtain

$$
\int_{B}\left|\nabla \tilde{\xi}_{j}\right|^{2} \tilde{G}^{\rho} \omega \leq \vartheta\left\|\tilde{\xi}_{j}\right\|_{\infty}\left(\tau_{j-1}+\left\|\tilde{\xi}_{j-1}\right\|_{\infty}\right)
$$

Using (3.10) to estimate $\left\|\tilde{\xi}_{j}\right\|_{\infty}$ in the above inequality, we get

$$
\tau_{j} \leq \vartheta\left(\tau_{j-1}+\left\|\tilde{\xi}_{j-1}\right\|_{\infty}\right), \quad j=1,2, \ldots
$$

The sum $\left\|\tilde{\xi}_{j}\right\|_{\infty}+\tau_{j}$ can thus be estimated as

$$
\left\|\tilde{\xi}_{j}\right\|_{\infty}+\tau_{j} \leq 2 \vartheta\left(\tau_{j-1}+\left\|\tilde{\xi}_{j-1}\right\|_{\infty}\right), \quad j=1,2, \ldots
$$

Observe that $\tau_{0}+\left\|\tilde{\xi}_{0}\right\|_{\infty} \leq\left(A_{0}\left\|f v^{-1}\right\|_{L_{v}^{p}(B)}\left\|\tilde{u}_{0}\right\|_{\infty}\right)^{1 / 2}+\left\|\tilde{u}_{0}\right\|_{\infty}$. But by Lemma 3.4, we recall $\left\|\tilde{u}_{0}\right\|_{\infty} \leq A_{0}\left\|f v^{-1}\right\|_{L_{v}^{p}(B)}$. Therefore from (3.10), and (3.11) one obtains by induction

$$
\left\|\tilde{\xi}_{j}\right\|_{\infty} \leq 2^{j-1} \vartheta^{j}\left(\tau_{0}+\left\|\tilde{\xi}_{0}\right\|_{\infty}\right) \leq(2 \vartheta)^{j} A_{0}\left\|f v^{-1}\right\|_{L_{v}^{p}(B)}, \quad j=1,2, \ldots
$$


An application of Cauchy-Schwarz inequality, and Lemma 3.1 leads us also, on using (1.1), to observe that

$$
\begin{aligned}
\int_{B}\left\langle A \nabla \tilde{\xi}_{j}, \nabla \tilde{\xi}_{j}\right\rangle= & a_{0}\left(\xi_{j}, \xi_{j}\right)=-\int_{B}\left(\mathbf{b} \cdot \nabla \tilde{\xi}_{j-1}+V \tilde{\xi}_{j-1}\right) \tilde{\xi}_{j} \\
\leq & \left(\int_{B}|\mathbf{b}|^{2} \omega^{-1} \tilde{\xi}_{j}^{2}\right)^{\frac{1}{2}} \cdot\left(\int_{B}\left|\nabla \tilde{\xi}_{j-1}\right|^{2} \omega\right)^{\frac{1}{2}} \\
& +\left(\int_{B}|V| \tilde{\xi}_{j}^{2}\right)^{\frac{1}{2}} \cdot\left(\int_{B}|V| \tilde{\xi}_{j-1}^{2}\right)^{\frac{1}{2}} \\
\leq & 2 \vartheta \int_{B}\left\langle A \nabla \tilde{\xi}_{j}, \nabla \tilde{\xi}_{j}\right\rangle+\frac{1}{2} \int_{B}\left|\nabla \tilde{\xi}_{j-1}\right|^{2} \omega .
\end{aligned}
$$

Therefore

$$
\beta_{j} \leq \frac{1}{\sqrt{2(1-2 \vartheta)}} \beta_{j-1}, \text { where } \beta_{j}:=\left(\int_{B}\left\langle A \nabla \tilde{\xi}_{j}, \nabla \tilde{\xi}_{j}\right\rangle\right)^{\frac{1}{2}}, \quad j=1,2, \ldots
$$

Thus

$$
\left\|\xi_{j}\right\|_{0}=\beta_{j} \leq\left(\frac{1}{\sqrt{2(1-2 \vartheta)}}\right)^{j} \beta_{0}=\left(\frac{1}{\sqrt{2(1-2 \vartheta)}}\right)^{j}\left\|\xi_{0}\right\|_{0} .
$$

Now, from (3.12) we observe that

$$
\left\|\tilde{u}_{m}-\tilde{u}_{k}\right\|_{\infty} \leq \sum_{j=k+1}^{m}\left\|\tilde{\xi}_{j}\right\|_{\infty} \leq A_{0}\left\|f v^{-1}\right\|_{L_{v}^{p}(B)} \sum_{j=k+1}^{m}(2 \vartheta)^{j-1}, \quad \text { for } m>k .
$$

Also, from (3.13) we obtain

$$
\left\|u_{m}-u_{k}\right\|_{0} \leq \sum_{j=k+1}^{m}\left\|\tilde{\xi}_{j}\right\|_{0} \leq\|\xi\|_{0} \sum_{j=k+1}^{m}\left(\frac{1}{\sqrt{2(1-2 \vartheta)}}\right)^{j}, \quad \text { for } m>k .
$$

Thus, if we further choose $r_{0}$ such $4 \vartheta(r)<1$ for $0<r \leq r_{0}$, then we conclude that $\left\{\tilde{u}_{m}\right\}$, and $\left\{u_{k}\right\}$ are Cauchy sequences in $L^{\infty}(B)$, and $H_{0}(B)$ respectively. So let us take $u_{*} \in H_{0}(B)$ such that $u_{m} \rightarrow u_{*}$ in $H_{0}(B)$. Now let $\varphi \in \operatorname{Lip}_{0}(B)$ be arbitrary. Then, we have

$$
D\left(u_{*}-u, \varphi\right)=D\left(u_{*}-u_{m}, \varphi\right)+D\left(u_{m}, \varphi\right)-D(u, \varphi) .
$$

But

$$
\begin{aligned}
D\left(u_{m}, \varphi\right)-D(u, \varphi) & =a_{0}\left(u_{m}, \varphi\right)+\int_{B}\left(\mathbf{b} \cdot\left(\nabla u_{m}\right) \varphi+V u_{m} \varphi\right)-\int_{B} f \varphi \\
& =a_{0}\left(u_{m}, \varphi\right)-a_{0}\left(u_{m+1}, \varphi\right)=a_{0}\left(u_{m}-u_{m+1}, \varphi\right) .
\end{aligned}
$$


Therefore, for $m \geq 1$

$$
\begin{aligned}
\left|D\left(u_{*}-u, \varphi\right)\right| & \lesssim\left\|u_{*}-u_{m}\right\|_{0}\|\varphi\|_{0}+\left|D\left(u_{m}, \varphi\right)-D(u, \varphi)\right| \\
& \lesssim\left\|u_{*}-u_{m}\right\|_{0}\|\varphi\|_{0}+\left|a_{0}\left(u_{m}-u_{m+1}, \varphi\right)\right| \\
& \lesssim\left(\left\|u_{*}-u_{m}\right\|_{0}+\left\|u_{m+1}-u_{m}\right\|_{0}\right)\|\varphi\|_{0} .
\end{aligned}
$$

Taking the limit as $m \rightarrow \infty$, we obtain $D\left(u_{*}-u, \varphi\right)=0$ for $\varphi \in \operatorname{Lip}_{0}(B)$. Since $\operatorname{Lip}_{0}(B)$ is dense in $H_{0}(B)$, and the bilinear form $D(.,$.$) is coercive$ on $H_{0}(B)$ we conclude that $u=u_{*}$. Since $\left\{\tilde{u}_{m}\right\}$ is a Cauchy sequence in $L^{\infty}(B)$, by uniqueness of limits we know that $\tilde{u}_{m} \rightarrow \tilde{u}$ in $L^{\infty}(B)$. But,

$$
\left\|\tilde{u}_{m}\right\|_{\infty} \leq \sum_{k=1}^{m}\left\|\tilde{\xi}_{k}\right\|_{\infty} \leq C A_{0}\left\|f v^{-1}\right\|_{L_{v}^{p}(B)} \sum_{k=1}^{m}(2 \vartheta)^{k-1} .
$$

Therefore, since

$$
\|\tilde{u}\|_{\infty} \leq\left\|\tilde{u}_{m}-\tilde{u}\right\|_{\infty}+C(r) A_{0}\left\|f v^{-1}\right\|_{L_{v}^{p}(B)},
$$

letting $m \rightarrow \infty$, and recalling the value of $A_{0}$, gives the desired estimation.

Remark 3.4. Let $f v^{-1} \in L_{v}^{p}(B)$ for some $p>\frac{\sigma}{\sigma-1}$. If $L^{*} u=f$ for $u \in$ $H_{0}(B)$, then $\tilde{L} u=f$, where $\tilde{L}:=-\operatorname{div}(A(x) \nabla)-\mathbf{b}(x) \cdot \nabla+(V-\operatorname{div} \mathbf{b})$. Therefore, if $|\mathbf{b}|^{2} \omega^{-1}$, $\operatorname{div} \mathbf{b}, V \in K$, then by the above theorem, we also have the estimate

$$
\|\tilde{u}\|_{L^{\infty}(B)} \leq C r^{2} \frac{v(B)^{\frac{1}{p^{\prime}}}}{\omega(B)}\left\|f v^{-1}\right\|_{L_{v}^{p}(B)},
$$

for some constant $C$.

For the rest of the paper we will require an additional condition on the coefficient $\mathbf{b}$ of the operator $L$. Thus, in addition to the condition (1.5) on the coefficients $\mathbf{b}$, and $V$ of $L$, we impose the following:

$$
\operatorname{div} \mathbf{b} \in K .
$$

Let $B \subset \subset B_{0}$ be a ball of radius sufficiently small that the bounded bilinear form (with $\mathbf{c} \equiv 0$ ) $D_{*}(u, \varphi)$ is coercive on $H_{0}(B)$. Let $y \in B$, and $\rho>0$ such that $B_{\rho}:=B_{\rho}(y) \subset B$. Since the map

$$
\varphi \mapsto f_{B_{\rho}} \tilde{\varphi} v
$$

is a continuous linear functional on $H_{0}(B)$, by Lax-Milgram theorem there is a unique $G^{\rho} \in H_{0}(B)$ such that

$$
D_{*}\left(G^{\rho}, \varphi\right)=f_{B_{\rho}} \tilde{\varphi} v, \quad \varphi \in H_{0}(B) .
$$


Following [3], we call $\tilde{G}^{\rho}$ the approximate Green function of $L$ on $B$ with pole $y$. Note that by Theorem 3.1 (see Remark 3.4), $\left\|\tilde{G}^{\rho}\right\|_{L^{\infty}(B)} \leq C$ for some constant $C$ depending on $\rho$, the pole of $\tilde{G}^{\rho}$, and $v$.

In the following Lemma, $B \subset B_{0}$ is a ball of radius so small that the bilinear form $D(.,$.$) is coercive.$

Lemma 3.6. Suppose $u \in H_{0}(B)$, and $D(u, \varphi) \geq 0$ whenever $0 \leq \varphi \in$ $\operatorname{Lip}_{0}(B)$. Then $u \geq 0$.

Proof. Let $u=\left\{u_{k}\right\}, u_{k} \in \operatorname{Lip}_{0}(B)$. Since $\nabla\left|u_{k}\right|=\left(\operatorname{sgn} u_{k}\right) \nabla u_{k}$ where $u_{k} \neq$ 0 , and $\left(\operatorname{sgn} u_{k}\right)\left|u_{k}\right|=u_{k}$, it follows that for each $k$

$$
D\left(u_{k},\left|u_{k}\right|\right)=D\left(\left|u_{k}\right|, u_{k}\right) \text {, and } D\left(\left|u_{k}\right|,\left|u_{k}\right|\right)=D\left(u_{k}, u_{k}\right) .
$$

Since $a_{0}\left(u_{k},\left|u_{k}\right|\right)=a_{0}\left(\left|u_{k}\right|, u_{k}\right)$ also, the sequence $\left\{\left|u_{k}\right|\right\}$ is bounded in $H_{0}(B)$, and thus a subsequence which we continue to write as $\left\{\left|u_{k}\right|\right\}$ converges weakly to some $v \in H_{0}(B)$. Since $\varphi \mapsto D(u, \varphi)$ is continuous on $H_{0}(B)$ and $D\left(u,\left|u_{k}\right|-u_{k}\right) \geq 0$ by hypothesis, it follows that

$$
0 \leq \lim _{k \rightarrow \infty} D\left(u,\left|u_{k}\right|-u_{k}\right)=D(u, v-u),
$$

so that $D(u, u) \leq D(u, v)$. Then $D(u, u)=\alpha D(u, v)$ for some $0<\alpha \leq 1$. Let us now observe that

$$
\begin{aligned}
0 \leq\left\|u-\alpha\left|u_{k}\right|\right\|_{0}^{2} & \lesssim D\left(u-\alpha\left|u_{k}\right|, u-\alpha\left|u_{k}\right|\right) \\
& =D(u, u)-2 \alpha D\left(u,\left|u_{k}\right|\right)+\alpha^{2} D\left(\left|u_{k}\right|,\left|u_{k}\right|\right) \\
& =D(u, u)-2 \alpha D\left(u,\left|u_{k}\right|\right)+\alpha^{2} D\left(u_{k}, u_{k}\right) .
\end{aligned}
$$

Taking the limit as $k \rightarrow \infty$, the last inequality reduces to

$$
\begin{aligned}
0 \leq \lim _{k \rightarrow \infty}\left\|u-\alpha\left|u_{k}\right|\right\|_{0}^{2} & \lesssim D(u, u)-2 \alpha D(u, v)+\alpha^{2} D(u, u) \\
& \leq D(u, u)-2 D(u, u)+\alpha^{2} D(u, u)=\left(\alpha^{2}-1\right) D(u, u) .
\end{aligned}
$$

Hence

$$
0 \leq \lim _{k \rightarrow \infty}\left\|u-\alpha\left|u_{k}\right|\right\|_{0}^{2} \lesssim\left(\alpha^{2}-1\right) D(u, u) \leq 0 .
$$

That is, $\alpha\left|u_{k}\right| \rightarrow u$ in $H_{0}(B)$ as $k \rightarrow \infty$, showing that $u$ is the limit in $H_{0}(B)$ of $\alpha\left|u_{k}\right| \geq 0$. From this, it also follows that $\alpha\left|u_{k}\right| \rightarrow \tilde{u}$ in $L_{v}^{2}$. But also $u_{k} \rightarrow \tilde{u}$ in $L_{v}^{2}(B)$. Therefore we must have $\alpha|\tilde{u}|=\tilde{u}$ a.e. on $B$. Thus $\alpha=1$, and hence $u=\left\{\left|u_{k}\right|\right\}$.

The following lemma will be useful.

Lemma 3.7. Let $B \subset \subset B_{0}$ be a ball of radius $r$, and $\tilde{G}^{\rho}$ be the approximate Green function of $L$ on $B$. There is $r_{0}>0$ such that if $0<r \leq r_{0}$, then

$$
\int_{B}\left(|\mathbf{b}|^{2} \omega^{-1}+|V|\right) \tilde{G}^{\rho} \leq C \eta\left(|\mathbf{b}|^{2} \omega^{-1}+|V|\right)(2 r),
$$

where $C$ is a constant independent of $\rho$ and the pole of $\tilde{G}^{\rho}$. 
Proof. Since

$$
\varphi \mapsto \int_{B}\left(|\mathbf{b}|^{2} \omega^{-1}+|V|\right) \tilde{\varphi}
$$

is a continuous linear functional on $H_{0}(B)$, by Lax-Milgram theorem let $\zeta \in H_{0}(B)$ be the unique solution of

$$
a_{0}(\zeta, \varphi)=\int_{B}\left(|\mathbf{b}|^{2} \omega^{-1}+|V|\right) \tilde{\varphi}, \quad \varphi \in H_{0}(B) .
$$

By Lemma 3.2, $\tilde{\zeta}(x) \lesssim \eta:=\eta\left(|\mathbf{b}|^{2} \omega^{-1}+V\right)(2 r)$ for a.e. $x \in B$. By Lemma 2.1, and Lemma 2.4 (or Remark 2.4), let us pick a sequence $\zeta_{k} \in$ $\operatorname{Lip}_{0}(B)$ such that $0 \leq \zeta_{k} \lesssim \eta$ a.e. on $B$. Let $\tilde{G}^{\rho}$ be the approximate Green function of $L$ on $B$. By Remark 3.4, $\tilde{G}^{\rho}$ is essentially bounded, and Lemma 3.6 shows $G^{\rho} \geq 0$. Thus by Lemma 2.4 (see Remark 2.4), we can pick a representative $G^{\rho}=\left\{G_{k}^{\rho}\right\}, G_{k}^{\rho} \in \operatorname{Lip}_{0}(B)$ such that for some constant $\mathrm{C}$ independent of $k$, we have $0 \leq G_{k}^{\rho} \leq C$ a.e. on $B$. Let us now observe that

$$
\begin{aligned}
& \int_{B}\left(|\mathbf{b}|^{2} \omega^{-1}+|V|\right) G_{k}^{\rho} \\
& =\delta_{k}+\int_{B}\left\langle A \nabla \zeta_{k}, \nabla G_{k}^{\rho}\right\rangle \\
& =\delta_{k}+\gamma_{k}-\int_{B}\left(\mathbf{b} \cdot\left(\nabla \zeta_{k}\right) G_{k}^{\rho}+V \zeta_{k} G_{k}^{\rho}\right)+f_{B_{\rho}} \zeta_{k} v \\
& \leq \delta_{k}+\gamma_{k}+\sqrt{\eta} \int_{B}|\mathbf{b}|^{2} \omega^{-1} G_{k}^{\rho}+\frac{1}{\sqrt{\eta}} \int_{B}\left\langle A \nabla \zeta_{k}, \nabla \zeta_{k}\right\rangle G_{k}^{\rho} \\
& +\eta \int_{B}|V| G_{k}^{\rho}+\eta .
\end{aligned}
$$

Here $\delta_{k}$ and $\gamma_{k}$ are given by

$$
\begin{aligned}
& \delta_{k}:=\int_{B}\left(|\mathbf{b}|^{2} \omega^{-1}+|V|\right) G_{k}^{\rho}-\int_{B}\left\langle A \nabla \zeta_{k}, \nabla G_{k}^{\rho}\right\rangle, \\
& \text { and } \gamma_{k}:=D_{*}\left(G_{k}^{\rho}, \zeta_{k}\right)-f_{B_{\rho}} \zeta_{k} v .
\end{aligned}
$$

By Remark 2.2, and Remark 3.2 respectively, we notice that $\delta_{k} \rightarrow 0$, and $\gamma_{k} \rightarrow 0$ as $k \rightarrow \infty$. We thus take the limit as $k \rightarrow \infty$. By Lemma 3.3, and the Lebesgue dominated convergence theorem, we obtain

$$
\begin{aligned}
& \int_{B}\left(|\mathbf{b}|^{2} \omega^{-1}+|V|\right) \tilde{G}^{\rho} \\
& \leq \sqrt{\eta} \int_{B}|\mathbf{b}|^{2} \omega^{-1} \tilde{G}^{\rho}+\frac{1}{\sqrt{\eta}} \int_{B}\langle A \nabla \tilde{\zeta}, \nabla \tilde{\zeta}\rangle \tilde{G}^{\rho}+\eta \int_{B}|V| \tilde{G}^{\rho}+\eta .
\end{aligned}
$$


As a result of (2.2), we see that $\left\{\zeta_{k} G_{k}^{\rho}\right\}$ is a bounded sequence in $H_{0}(B)$. Therefore, we pick a subsequence still denoted by $\left\{\zeta_{k} G_{k}^{\rho}\right\}$ that converges weakly in $H_{0}(B)$, and such that $\zeta_{k} G_{k}^{\rho} \rightarrow \tilde{\zeta} \tilde{G}^{\rho}$ pointwise a.e. on $B$. Using this subsequence we have

$$
\begin{aligned}
\int_{B}\left\langle A \nabla \zeta_{k}, \nabla \zeta_{k}\right\rangle G_{k}^{\rho} & =\int_{B}\left\langle A \nabla \zeta_{k}, \nabla\left(\zeta_{k} G_{k}^{\rho}\right)\right\rangle-\frac{1}{2} \int_{B}\left\langle A \nabla G_{k}^{\rho}, \nabla \zeta_{k}^{2}\right\rangle \\
& =\delta_{k}+\int_{B}\left(|\mathbf{b}|^{2} \omega^{-1}+|V|\right) G_{k}^{\rho} \zeta_{k} \\
& +\frac{1}{2}\left(\int_{B}\left(\mathbf{b} \cdot\left(\nabla \zeta_{k}^{2}\right) G_{k}^{\rho}+V G_{k}^{\rho} \zeta_{k}^{2}\right)+\gamma_{k}-f_{B_{\rho}} \zeta_{k}^{2} v\right) \\
& \leq \delta_{k}+\frac{1}{2} \gamma_{k}+\eta \int_{B}\left(|\mathbf{b}|^{2} \omega^{-1}+|V|\right) G_{k}^{\rho} \\
& +\frac{1}{2}\left(\eta^{2} \int_{B}\left(|\mathbf{b}|^{2} \omega^{-1}+|V|\right) G_{k}^{\rho}+\int_{B}\left\langle A \nabla \zeta_{k}, \nabla \zeta_{k}\right\rangle G_{k}^{\rho}\right),
\end{aligned}
$$

where

$$
\begin{aligned}
& \delta_{k}:=a_{0}\left(\zeta_{k}, \zeta_{k} G_{k}^{\rho}\right)-\int_{B}\left(|\mathbf{b}|^{2} \omega^{-1}+|V|\right) G_{k}^{\rho} \zeta_{k}, \\
& \text { and } \gamma_{k}:=-D_{*}\left(G_{k}^{\rho}, \zeta_{k}^{2}\right)+f_{B_{\rho}} \zeta_{k}^{2} v .
\end{aligned}
$$

Again by Remark 2.2, and Remark 3.2 respectively, we see that $\delta_{k} \rightarrow 0$, and $\gamma_{k} \rightarrow 0$ as $k \rightarrow \infty$. We thus take the limit as $k \rightarrow \infty$. By Lemma 3.3, and the Lebesgue dominated convergence theorem, we obtain

$$
\begin{aligned}
\int_{B}\langle A \nabla \tilde{\zeta}, \nabla \tilde{\zeta}\rangle \tilde{G}^{\rho} & \leq \eta \int_{B}\left(|\mathbf{b}|^{2} \omega^{-1}+|V|\right) \tilde{G}^{\rho} \\
& +\frac{1}{2}\left(\eta^{2} \int_{B}\left(|\mathbf{b}|^{2} \omega^{-1}+|V|\right) \tilde{G}^{\rho}+\int_{B}\langle A \nabla \tilde{\zeta}, \nabla \tilde{\zeta}\rangle \tilde{G}^{\rho}\right) .
\end{aligned}
$$

Putting this last inequality back into (3.15), we see that there is $r_{0}$ such that for all $0<r \leq r_{0}$,

$$
\int_{B}\left(|\mathbf{b}|^{2} \omega^{-1}+|V|\right) \tilde{G}^{\rho} \leq C \eta\left(|\mathbf{b}|^{2} \omega^{-1}+|V|\right)(2 r),
$$

as required.

We now have all the needed ingredients to demonstrate the uniform integrability of the approximate Green functions of $L$. We use the methods in [3] (see also [5], [8]) to prove the integrability theorem.

Theorem 3.2. Let $B \subset \subset B_{0}$ be a ball of radius $r$. Suppose $\tilde{G}^{\rho}$ is the approximate Green function of $L$ on $B$, where we assume that the coefficients 
of $L$ satisfy the conditions (1.5) and (3.14). Then for $0<p<\sigma$ there is a positive constant, independent of $\rho$ and the pole, such that

$$
\left(f_{B}\left(\tilde{G}^{\rho}\right)^{p} v\right)^{\frac{1}{p}} \leq C \frac{r^{2}}{\omega(B)},
$$

when $r$ is sufficiently small.

Proof. Let $\tilde{G}^{\rho}$ be the approximate Green function of $L$ on $B$. By Lemma 3.6, $G^{\rho} \geq 0$ on $B$. Therefore we pick a representative $G^{\rho}=\left\{G_{k}^{\rho}\right\}, G_{k}^{\rho} \in \operatorname{Lip}_{0}(B)$ such that $G_{k}^{\rho} \geq 0$.

Now, for $t>0$, let us define

$$
\varphi_{k}:=\left[\frac{1}{t}-\frac{1}{G_{k}^{\rho}}\right]^{+}=\left[\frac{1}{t}-\frac{1}{G_{k}^{\rho}}\right] \chi_{\left\{G_{k}^{\rho}>t\right\}} .
$$

Then $\varphi_{k} \in \operatorname{Lip}_{0}(B)$, and

$$
\nabla \varphi_{k}=\frac{\nabla G_{k}^{\rho}}{\left(G_{k}^{\rho}\right)^{2}} \chi_{\left\{G_{k}^{\rho}>t\right\}}
$$

Since $\left\|\varphi_{k}\right\|_{0}^{2} \leq\left\|G_{k}^{\rho}\right\|_{0}^{2} / t^{4}$, we can pick a subsequence, still denoted by $\left\{\varphi_{k}\right\}$ that converges weakly in $H_{0}(B)$. With such subsequence, we observe that

$$
\int_{B}\left\langle A \nabla G_{k}^{\rho}, \nabla \varphi_{k}\right\rangle=\delta_{k}-\int_{B}\left(\mathbf{b} \cdot \nabla \varphi_{k}+V \varphi_{k}\right) G_{k}^{\rho}+f_{B_{\rho}} \varphi_{k} v
$$

where

$$
\delta_{k}:=D_{*}\left(G_{k}^{\rho}, \varphi_{k}\right)-f_{B_{\rho}} \varphi_{k} v .
$$

Using the Cauchy-Schwarz inequality, and noting that $\varphi_{k} \leq 1 / t$ on $B$, we estimate

$$
\begin{aligned}
\int_{B}\left(\mathbf{b} \cdot \nabla \varphi_{k}+V \varphi_{k}\right) G_{k}^{\rho} & \leq \frac{1}{2 t} \int_{B}|\mathbf{b}|^{2} \omega^{-1} G_{k}^{\rho}+\frac{t}{2} \int_{B}\left|\nabla \varphi_{k}\right|^{2} G_{k}^{\rho} \omega+\frac{1}{t} \int|V| G_{k}^{\rho} \\
& \leq \frac{1}{t} \int_{B}\left(|\mathbf{b}|^{2} \omega^{-1}+|V|\right) G_{k}^{\rho}+\frac{t}{2} \int_{\left\{G_{k}^{\rho}>t\right\}} \frac{\left|\nabla G_{k}^{\rho}\right|^{2}}{\left(G_{k}^{\rho}\right)^{4}} G_{k}^{\rho} \omega \\
& \leq \frac{1}{t} \int_{B}\left(|\mathbf{b}|^{2} \omega^{-1}+|V|\right) G_{k}^{\rho}+\frac{1}{2} \int_{\left\{G_{k}^{\rho}>t\right\}} \frac{\left|\nabla G_{k}^{\rho}\right|^{2}}{\left(G_{k}^{\rho}\right)^{2}} \omega \\
& \leq \frac{1}{t} \int_{B}\left(|\mathbf{b}|^{2} \omega^{-1}+|V|\right) G_{k}^{\rho}+\frac{1}{2} \int_{B}\left\langle A \nabla G_{k}^{\rho}, \nabla \varphi_{k}\right\rangle .
\end{aligned}
$$

Taking this last estimation, and using again the fact that $\varphi_{k} \leq 1 / t$ on $B$ we get from (3.16) that

$$
\int_{B}\left\langle A \nabla G_{k}^{\rho}, \nabla \varphi_{k}\right\rangle \leq 2\left|\delta_{k}\right|+\frac{2}{t} \int_{B}\left(|\mathbf{b}|^{2} \omega^{-1}+|V|\right) G_{k}^{\rho}+\frac{2}{t} .
$$


We now take the limit as $k \rightarrow \infty$. By Remark 3.2 we know that $\delta_{k} \rightarrow 0$ as $k \rightarrow \infty$. Using this fact and applying Lemma 3.7, we get

$$
\begin{aligned}
\lim _{k \rightarrow \infty} \int_{B}\left\langle A \nabla G_{k}^{\rho}, \nabla \varphi_{k}\right\rangle & \leq \frac{2}{t} \int_{B}\left(|\mathbf{b}|^{2} \omega^{-1}+|V|\right) \tilde{G}^{\rho}+\frac{2}{t} \\
& \leq \frac{2}{t}\left(1+\eta\left(|\mathbf{b}|^{2} \omega^{-1}+V\right)(3 r)\right) .
\end{aligned}
$$

Hence by the degeneracy condition, we obtain the inequality

$$
\limsup _{k \rightarrow 0} \int_{\left\{G_{k}^{\rho}>t\right\}} \frac{\left|\nabla G_{k}^{\rho}\right|^{2}}{\left(G_{k}^{\rho}\right)^{2}} \omega \leq \frac{2}{t}\left(1+\eta\left(|\mathbf{b}|^{2} \omega^{-1}+V\right)(3 r)\right) .
$$

Now let

$$
\psi_{k}:=\left[\log G_{k}^{\rho}-\log t\right]^{+}=\left[\log G_{k}^{\rho}-\log t\right]_{\chi_{\left\{G_{k}^{\rho}>t\right\}}} .
$$

Then $\psi_{k} \in H_{0}(B)$, and $\nabla \psi_{k}=\left(\frac{\nabla G_{k}^{\rho}}{G_{k}^{\rho}}\right) \chi_{\left\{G_{k}^{\rho}>t\right\}}$. Therefore, for sufficiently small $r$ the last inequality now reads

$$
\limsup _{k \rightarrow \infty} \int_{B}\left|\nabla \psi_{k}\right|^{2} \omega \leq \frac{C}{t}
$$

and hence by Sobolev inequality (2.1), we get

$$
\limsup _{k \rightarrow \infty}\left(\frac{1}{v(B)} \int_{B \cap\left\{G_{k}^{\rho}>t\right\}} \log \left(\frac{G_{k}^{\rho}}{t}\right)^{q} v\right)^{\frac{2}{q}} \leq C \frac{r^{2}}{\omega(B)} \frac{C}{t} .
$$

Restricting the integration to $\left\{G_{k}^{\rho}>2 t\right\}$, we get

$$
(\log 2)^{2} \limsup _{k \rightarrow \infty}\left(\frac{v\left(\left\{G_{k}^{\rho}>2 t\right\}\right)}{v(B)}\right)^{\frac{2}{q}} \leq C \frac{r^{2}}{\omega(B)} \frac{1}{t} .
$$

The above inequality remains valid if we replace $2 t$ by $t$. Also by using further subsequence if necessary, we may assume that $G_{k}^{\rho} \rightarrow \tilde{G}^{\rho}$ pointwise a.e. on $B$. Thus $\chi_{\left\{\tilde{G}^{\rho}>t\right\}} \leq \liminf \chi_{\left\{G_{k}^{\rho}>t\right\}}$ a.e., and by Fatou's lemma,

$$
v\left(\left\{\tilde{G}^{\rho}>t\right\}\right) \leq \liminf _{k \rightarrow \infty} v\left(\left\{G_{k}^{\rho}>t\right\}\right) .
$$

Therefore we obtain,

$$
v\left(\left\{\tilde{G}^{\rho}>t\right\}\right) \leq C\left(\frac{r^{2}}{\omega(B)}\right)^{\sigma} \frac{1}{t^{\sigma}} v(B) .
$$

The theorem then follows from this estimate, and the formula

$$
\begin{aligned}
\int_{B}\left(\tilde{G}^{\rho}\right)^{p} & =p \int_{0}^{\infty} t^{p-1} v\left(\left\{\tilde{G}^{\rho}>t\right\}\right) \\
& \leq p v(B) \int_{0}^{A} t^{p-1}+p \int_{A}^{\infty} t^{p-1} v\left(\left\{\tilde{G}^{\rho}>t\right\}\right),
\end{aligned}
$$

where $A=r^{2} / \omega(B)$. 
Acknowledgments. I would like to record my indebtness to Professor C.E. Gutierrez for his constant encouragement and his invaluable advice throughout the preparation of this paper. I would also like to thank the referee for the timely and careful reading of the manuscript.

\section{References}

[1] A. Aizenman and B. Simon, Brownian motion and Harnack's inequality for Schrödinger operators, Comm. Pure Appl. Math., 35 (1982), 209-273, MR 84a:35062, Zbl 475.60063.

[2] S. Chanillo and R.L. Wheeden, Weighted Poncarè and Sobolev inequalities and estimates for the Peano maximal function, Amer. J. Math., 107 (1985), 1191-1226, MR 87f:42045, Zbl 575.42026.

[3] __ Existence and estimates of Green's function for degenerate elliptic equations, Scuola Norm. Sup., Pisa, XV. Fasc. II (1989), 309-340, MR 91b:35045.

[4] F. Chiarenza, E. Fabes and N. Garofalo, Harnack's inequality for Schrödinger operators and continuity of solutions, Proc. Amer. Math. Soc., 98 (1986), 415-425, MR 88a:35037, Zbl 626.35022.

[5] M. Gruter and K.O. Widman, The Green function for uniformly elliptic equations, Manuscripta Math., 37 (1982), 303-342, MR 83h:35033, Zbl 485.35031.

[6] C.E. Gutierrez, Harnack's inequality for degenerate Schrödinger operators, Trans. Amer. Math. Soc., 312 (1989), 403-419, MR 90g:35062, Zbl 685.35020.

[7] K. Kurata, Continuity and Harnack's inequality for solutions of elliptic partial differential equations of second order, Indiana U. Math. J., 43(2) (1994), 411-440, MR 95f:35054, Zbl 805.35017.

[8] G. Lu, Existence and size estimates for the Green's functions of differential operators constructed from degenerate vector fields, Comm. PDE, 17 (1992), 1213-1251, MR 93i:35030, Zbl 798.35002.

[9] J.O. Strömberg and A. Torchinsky, Weighted Hardy spaces, in 'Weighted Hardy Spaces' (Lecture notes in Mathematics), 1381, Springer Verlag, New York, (1989), 83-128, MR 90j:42053, Zbl 676.42021.

Received September 10, 1999 and revised February 15, 2000. This research was partially supported by Kuwait University grant SM-137.

Temple University

Philadelphia, PA 19122

E-mail address: ahmed@math.temple.edu 